\title{
Electro-thermal Modelling of Monolithic and Hybrid Microwave and Millimeter Wave IC's
}

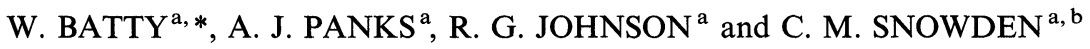 \\ ${ }^{a}$ Institute of Microwaves and Photonics, School of Electronic and Electrical Engineering, \\ The University of Leeds, Leeds LS2 9JT, UK; ${ }^{\mathrm{b}}$ Filtronic plc, The Waterfront, \\ Salts Mill Road, Shipley BD18 3TT, UK
}

(Received 16 December 1998; In final form 14 December 1999)

\begin{abstract}
The first completely physical electro-thermal model is presented that is capable of describing the large signal performance of MESFET- and HEMT-based, high power microwave and millimeter wave monolithic and hybrid ICs, on timescales suitable for CAD. The model includes the effects of self-heating and mutual thermal interaction on active device performance with full treatment of all thermal non linearities. The electrical description is provided by the rapid quasi-2D Leeds Physical Model and the steady-state global thermal description is provided by a highly accurate and computationally inexpensive analytical thermal resistance matrix approach. The order of the global thermal resistance matrix describing 3-dimensional heat flow in complex systems, is shown to be determined purely by the number of active device elements, not the level of internal device structure. Thermal updates in the necessarily iterative, fully coupled electro-thermal solution, therefore reduce to small matrix multiplications implying orders of magnitude speed-up compared to the use of full numerical thermal solutions capable of comparable levels of detail and accuracy.
\end{abstract}

Keywords: Electro-thermal, thermal, modelling, MMIC, power transistor

\section{INTRODUCTION}

The importance of thermal effects in the description of high power device and circuit performance has long been recognised, and much work has been done in modelling the effects of self-heating and mutual thermal interaction on device and circuit behaviour [1-48]. If electro-thermal models are to be of value for predictive electro-thermal design studies, based purely on specified material and structural information rather than on prior experimental characterisation, then fully physical (as opposed to equivalent circuit or physics-based) coupled models are required. However, although physical thermal simulations have been performed for complex device structures, relatively little work has been presented describing explicit coupling of physical thermal and large signal electrical models.

${ }^{*}$ Corresponding author. Tel.: + 44113 2332089, Fax: +44 113 2332032, e-mail: w.batty@elec-eng.leeds.ac.uk 
The reason for the absence of such coupled calculations arises from three main sources. Firstly, fully physical device models with large signal electrical capability are still not commonplace. Secondly, although the basic thermal issues are well understood, thermal solvers available in other areas of study, such as mechanical engineering, are not readily adaptable to electronic engineering problems. Thirdly, the coupled electro-thermal problem is intrinsically non linear implying the need for iterative solutions, and existing physical thermal and electrical solvers are generally too slow to generate an iterative coupled solution sufficiently fast for electro-thermal design studies.

Of the existing thermal models with the potential for coupled electro-thermal simulations, that of Bonani et al., for steady-state simulation of heatsink mounted MMICs is most comprehen sive $[1-3]$. This model describes arbitrary surface metallisation, vias and partial substrate thinning for multi-finger power FETs and MMICs. Thermal solutions based on this hybrid Green's function finite element approach typically take $\sim 1$ hour on a medium-sized workstation [3]. Webb has performed thermal simulations for both steady-state and thermal transient cases [4-8], and typical steady-state thermal simulations take $\sim 1 / 2$ hour [8] on a PC using an optimised finite difference scheme. Dorkel et al., have performed steady-state and transient thermal simulations taking just a few minutes but these were for simplified multilayer structures, based on a 2-port network (or $2 \times 2$ transfer matrix) formulation [9]. All of these calculations assumed adiabatic sur face boundary conditions. Newtonian boundary conditions have been imposed for use at the cir cuit board level where surface areas are large, for instance by Ellison who performed analytical Green's function calculations for simple multilayer structures $[10,11]$. Electro-thermal simulations have been performed by Anholt for HBTs, MESFETs and HEMTs [12-15] and compared against simple analytical expressions for thermal resistances [15]. Ghione and Naldi performed fully physical, self-consistent electro-thermal calculations for MESFETs in MMICs [16]. The boundary conditions for their physical model were provided by a thermal resistance formulation, with the thermal resistances obtained approximately using a conformal mapping technique.

It has been stated repeatedly [2,9] that the thermal resistance method is unsuitable for accurate description of detailed device structures, but this assertion depends on a particular conception of the thermal resistance approach as being fundamentally approximate. This paper shows that the thermal resistance matrix concept [16-19] can be developed as an essentially analytically exact description of solutions of the heat diffusion equation at selected points within a complex 3dimensional volume. Temperature updates in the necessarily iterative coupled electro-thermal solution, based on this resistance matrix approach, reduce to small matrix multiplications with the order of the matrices determined solely by the number of active device elements. Such solutions are therefore orders of magnitude faster than solutions based on finite element or finite difference thermal descriptions, whilst achieving the same levels of detail and accuracy.

The thermal resistance matrix description presented here is applicable from individual MMICs through hybrid MICs, RFICs and MMIC grid arrays to circuit board level, where the number of active device elements is $\sim 10$ to several $\times 10^{3}$. Electro-thermal simulations for VLSI design have been presented previously [20]. However, use of the thermal resistance matrix approach presented here, in an unmodified form, with the order of the dense thermal resistance matrix given by the number of individual active devices typical in VLSI or ULSI, would clearly be of no advantage compared to standard numerical solutions of the heat diffusion equation or to sparse matrix thermal network formulations. The thermal resistance matrix description given in this paper is therefore not intended to apply directly to the VLSI case.

The global thermal resistance matrix model described here is given in the context of coupled 
electro-thermal modelling. However, it is applicable to any thermal problem that can be well described by the temperatures in the vicinities of a relatively small number of localised regions, rather than requiring temperature information throughout the whole body of a complex volume. Use of the Kirchhoff transformation, combined with use of the resistance matrix for (generally non linear) interface matching of analytical subsystem solutions, is more generally applicable to any physical system described by Laplace's equation

$$
\nabla \cdot[g(\psi) \nabla \psi]=0
$$

providing the system can be sufficiently accurately represented by rectangular subsystems, with piecewise discretisation of $\psi$ and its derivative, $\psi^{\prime}$, at the interfaces.

The boundary element method uses a Green's function technique to replace 3-dimensional numerical finite element or finite difference descriptions, given by large sparse matrices, with a 2-dimensional surface description given by smaller dense matrices. In a similar fashion the analytical thermal resistance matrix approach reduces the thermal description to that of very small dense matrices, describing only limited 2-dimensional regions at surfaces and discretised interfaces by direct solution of the heat diffusion equation. All explicit reference to redundant temperature information at discretised volume and surface nodes is eliminated. This thermal resistance matrix approach can equally be viewed as a generalised finite element or finite volume approach, in which the finite elements are not primitive volumes with temperatures between nodes described by low order polynomial interpolation, but complete thermal subsystems with internal temperatures given by full analytical solutions of the heat diffusion equation. Construction of a global thermal resistance matrix then consists of matrix manipulations on resistance matrices given by simple analytical expressions for thermal subsystems.

A key feature of this thermal resistance matrix approach is that the global thermal resistance matrix only has to be evaluated once, for the thermal steady-state case treated here, prior to the coupled electro-thermal solution. Construction time for the thermal resistance matrices has no impact on coupled electro-thermal run time. The global thermal resistance matrix is itself constructed rapidly by simple matrix manipulations on analytical expressions for thermal subelements, such as MMIC dies or sections of surface metallisation, each evaluated purely in terms of subsystem layout and material parameters. The global resistance matrix describes totally arbitrary device layouts (based on simple rectangular structures) in 3-dimensions and once constructed can be used repeatedly in coupled electro-thermal simulations.

Models containing descriptions of self-heating effects on the performance of a range of devices have been described previously, e.g. [17, 21-23]. The global thermal model presented here is intended to be readily compatible with any thermally self-consistent device model, without requiring core changes. It should then supply a fully physical and highly accurate description of mutual thermal interaction between active devices at chip level and above.

The paper provides an overview of the analytical thermal resistance matrix approach, demonstrating how it can achieve the same level of accuracy and detail as finite element or finite difference descriptions such as those of Bonani or Webb, whilst reducing the global thermal description to small dense matrix multiplications. This reduction implies rapid temperature updates in the coupled electro-thermal model allowing fully physical solution on timescales suitable for CAD. The global thermal description combines analytical solutions of the heat diffusion equation for thermal subsystems with hierarchical interface matching of thermal resistance matrices for complex 3-dimensional systems. It is shown that this approach allows accurate treatment of all thermal non linearities arising from temperature dependent material constants and surface fluxes.

The paper is structured as follows. The next section contains a brief outline of the Leeds 
Physical Model (LPM) which provides the large signal active device description required in the coupled electro-thermal model. This is followed by a statement of the coupled electro-thermal problem and an indication of the key issues in the global thermal description. The motivation for the thermal resistance matrix approach to the global thermal problem is then indicated. This is followed by description of the analytical thermal steady-state solutions for the MMIC and for other subsystem components. Simple analytical expressions are presented for the thermal resistance matrices of entire MMICs bearing several multi-finger power transistors. A linear 'radiation' boundary condition is imposed to describe convective and radiative surface losses from large area substrates. A hierarchical approach is presented for the resistance matrix description of multilayered structures such as MMICs with superstructure, e.g., surface metallisation and air bridges, or for multiple MMICs mounted on a common substrate. A double Fourier series finite element technique is outlined for the construction of the thermal resistance matrices of ICs with totally arbitrary surface metallisation and full 3-dimensional heat flow. An original analytical solution is presented for the 3-dimensional temperature distribution and corresponding thermal resistance matrix in a hybrid MIC or MMIC with any number of arbitrarily placed vias of arbitrary crosssection, or with partial substrate thinning. Finally, the paper is summarised and conclusions drawn.

\section{THE LEEDS PHYSICAL MODEL}

The Leeds Physical Model [22,49-51] is a quasi2D physical model of MESFETs and HEMTs including the effects of self-heating. It is a sophisticated CAD tool and has been released commercially as part of HP-EEsof's MDS software.

The quasi-2D approximation is based on the observation, from full 2-dimensional simulations, that carrier transport is essentially 1-dimensional and driven by the component of electric field along the device channel. The model solves a set of hydrodynamic equations obtained from moments of the Boltzmann equation:

$$
\begin{aligned}
\frac{\partial n}{\partial t}+\nabla \cdot(n v)= & 0 \\
\frac{\partial v}{\partial t}+v \cdot \nabla v= & \frac{q}{m^{*}} E-\frac{2}{3 m^{*} n} \nabla(n w) \\
& +\frac{1}{3 n} \nabla\left(n v^{2}\right)-\frac{v}{\tau_{p}}, \\
\frac{\partial w}{\partial t}+v \cdot \nabla w= & q v \cdot E-\frac{2}{3 n} \nabla \cdot\left[n v\left(w-\frac{m^{*}}{2} v^{2}\right)\right] \\
& -\frac{1}{n} \nabla \cdot Q-\frac{w-w_{0}}{\tau_{w}}, \\
c_{L} \rho_{L} \frac{\partial T}{\partial t}= & \nabla \cdot\left(\kappa_{L} \nabla T\right)+J \cdot E,
\end{aligned}
$$

in the direction parallel to the heterointerface (suitably reduced for rapid solution) [49]. Here $n$ is carrier density, $v$ is velocity, $w$ is energy, $m^{*}$ is effective mass, $E$ is electric field, $\tau_{p}, \tau_{w}$ are relaxation lifetimes, $T$ is lattice temperature, $c_{L}$ is heat capacity, $\rho_{L}$ is density, $t$ is time, $\kappa_{L}$ is thermal conductivity and $J$ is current density. This energy transport model then consists of continuity, momentum conservation, energy conservation, and heat diffusion equations.

The LPM solves Poisson and Schrodinger equations self-consistently in the direction normal to the heterointerface, to describe charge control, Figure 1. The charge control information is calculated prior to calculation of in-plane transport and is stored as a look-up table.

The quasi-2D model includes a full description of the device cross-section, by describing charge conservation in the vicinity of the device channel via a series of Gaussian boxes, Figure 2 .

The Leeds Physical Model incorporates the effects of temperature on device performance by use of a temperature dependent low field mobility,

$$
\mu=\mu\left(T_{o}\right)\left(\frac{T_{o}}{T}\right)^{n}
$$




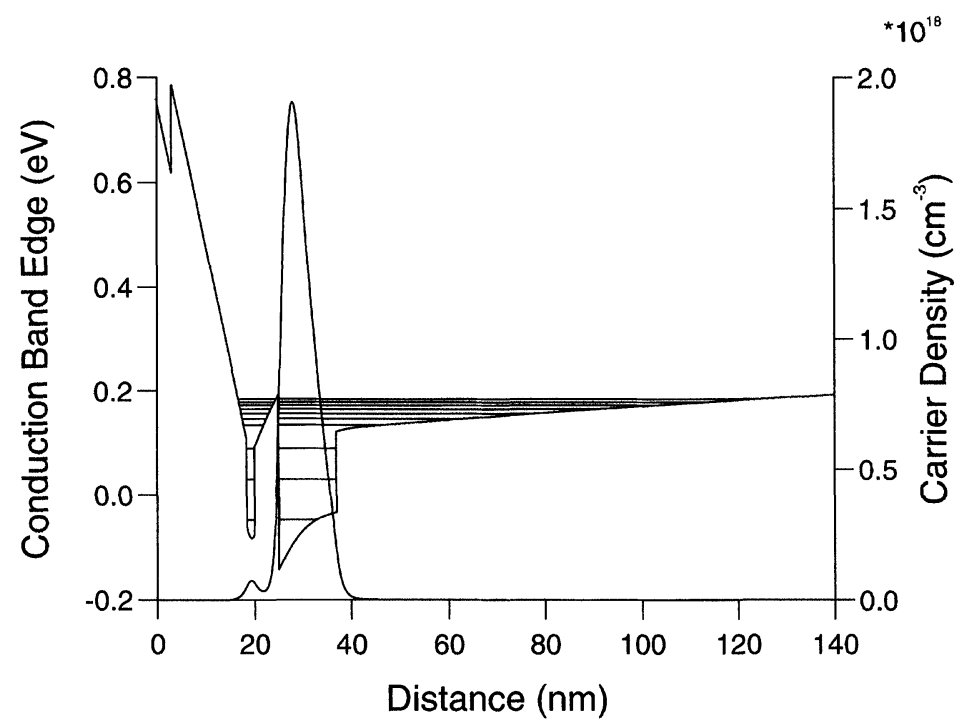

FIGURE 1 Self-consistent Schrodinger-Poisson solution from the LPM for HEMT band-edge profile, subband structure, and carrier density normal to the heterointerface [51].

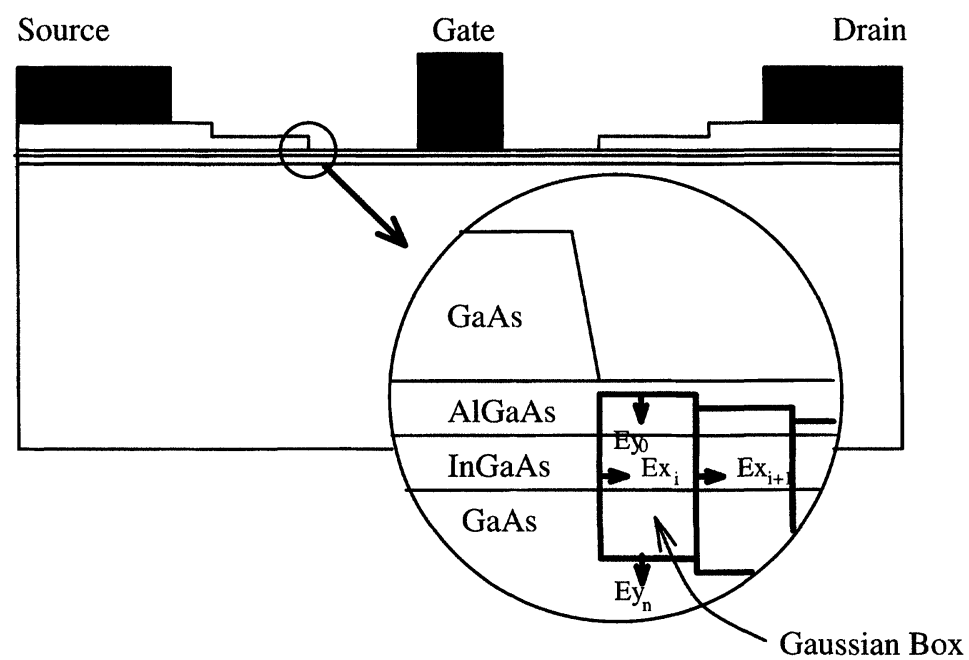

FIGURE 2 Typical FET cross-section and layer structure illustrating Gaussian boxes used in the LPM to model the 2-dimensional profile [22].

where $T$ is the device channel temperature and $n=2.3$ [52]. Velocity-field characteristics are obtained from Monte Carlo simulations and stored as a simple parameterisation.

The LPM provides a physical large signal description of device performance, as illustrated by Figure 3, which shows calculated time and frequency domain response to a sinusoidal large signal input [53]. Output waveform distortion and generation of harmonics differing from the fundamental are clear.

The LPM forms the core of the coupled electrothermal solution presented in this paper. The coupled electro-thermal problem is now described. 


\section{$V_{D S}$ Output Waveform}

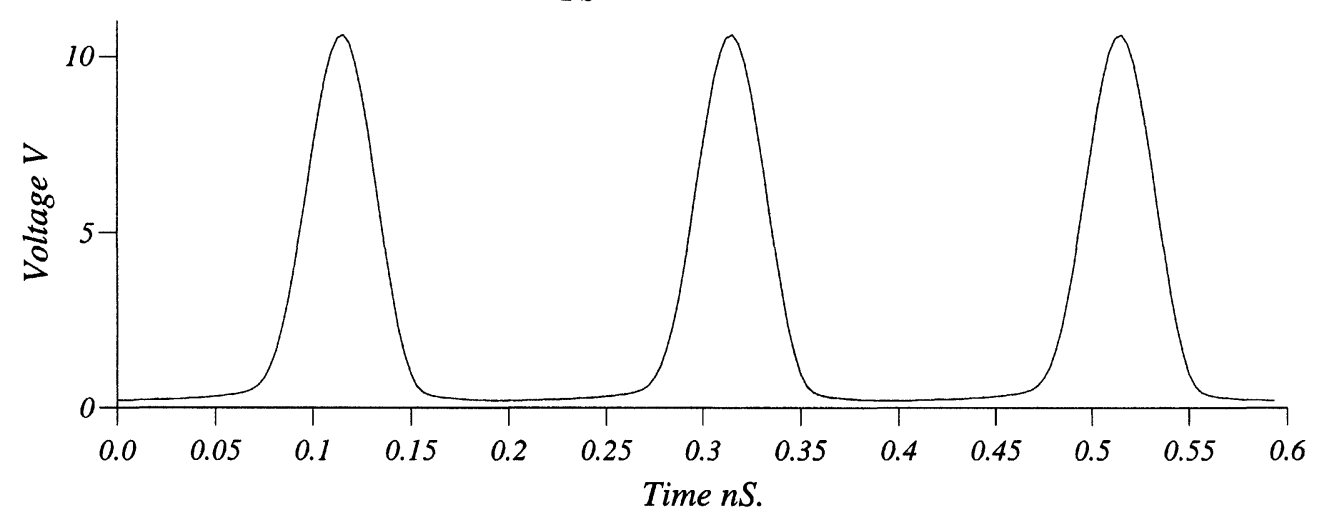

DFT.

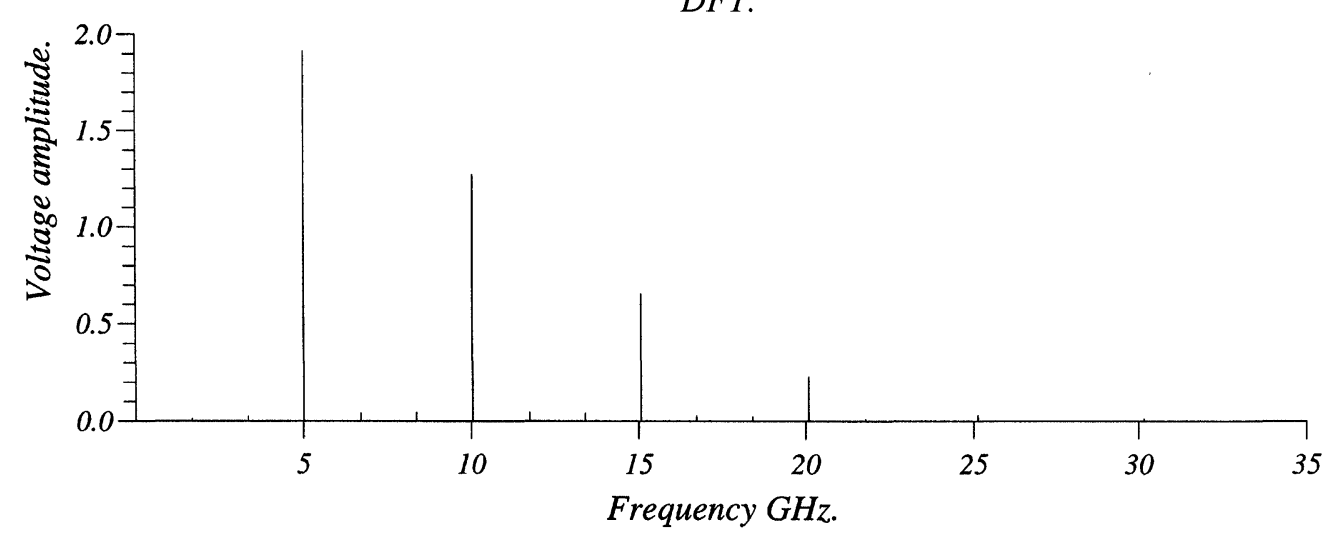

FIGURE 3 LPM time and frequency domain response to a sinusoidal $5 \mathrm{GHz}, 0.45 \mathrm{~V}$ input signal [53].

\section{THE COUPLED ELECTRO-THERMAL PROBLEM}

The non linear coupled electro-thermal problem can be described by the equation

$$
\theta_{i}\left(P_{1}, \ldots, P_{M}\right)-\theta_{i}\left(P_{i}\right)=0 \text { for } i=1, \ldots, M
$$

for self-consistent solution of active device temperatures $\theta_{i}$ and power dissipations $P_{i}$, in device channels $i=1, \ldots, M$.

The first term states that the channel temperature of the $i$ th active device is a (non linear) function of the power dissipations $P_{j}$ of all heat dissipating elements, $j=1, \ldots, M$, as described by the global thermal model. The second term states that the $i$ th channel temperature has a unique relation to the corresponding power dissipation $P_{i}$ of the $i$ th active element as determined by the LPM.

Simultaneous imposition of the non linear description of heat flow through the MMIC or hybrid MIC (with surface heat loss), and the non linear electrical description of transistor action, determine uniquely the temperatures and power dissipations in the vicinities of the active device channels. These in turn, determine full electrical solutions, DC or large signal RF. The above description applies equally well to both the 
thermal steady-state and to the time-dependent case in Laplace transform space.

Details of time-dependent electrical behaviour are decoupled from thermal behaviour by the fact that the inverse lattice thermal time constant is orders of magnitude different from $\mathrm{GHz}$ electrical frequencies. The large signal RF case is therefore treated by time averaging the electrical signal (to describe effects such as AC cooling). Only the steady-state thermal case is treated here. Thermal resistance matrix treatment of thermal transients due to turn-on, or due to low frequency pulsed operation, will be described elsewhere [24].

\subsection{Coupling the Leeds Physical Model}

The manner in which the LPM enters the coupled solution is easily illustrated for the DC case. The LPM is invoked as a simple subroutine call for each active device element

$$
I_{D S}=I_{D S}\left(V_{D S}, V_{G S} ; T\right) .
$$

This device element will typically be a single source-gate-drain subunit, or a fraction of such a unit divided along the gate width.

Supplying bias point $\left(V_{D S}, V_{G S}\right)$, where $V_{D S}$ is drain-source voltage and $V_{G S}$ is gate-source voltage, and with active device channel temperature given by $T$ (assumed uniform along the channel for simplicity of description), the LPM returns drain-source current $I_{D S}$. The active device power dissipation $P$ is then given by the approximate relation,

$$
P \approx I_{D S} V_{D S}
$$

$P$ is the time average value in the time dependent case.

A single, uniform channel temperature is assumed in the current coupled electro-thermal solution, but this is not a necessary condition for solution. The LPM returns the power dissipation $P=E \cdot J$ as a function of field, $E$, and current density, $J$, along the device channel. By subdividing the gate along its length the resistance matrix approach can describe a varying channel temperature, with consequent improvement in transistor breakdown modelling [23] and without the need to artificially displace the gate in order to describe correctly the position of the peak temperature in the vicinity of a gate finger. As the LPM returns $E \cdot J$, the extent of the device heating is determined accurately by the physical model. No approximation is required to provide an effective gate length for comparison against experiment [16]. The position dependent power dissipation in the LPM is finely discretised, but the discretisation used for the thermal description, along the source-gatedrain length, can be much coarser [4]. The resulting temperature distribution obtained from the corresponding analytical thermal solution is continuous, with the only approximation being the averaging of the power densities over the source-gate-drain sublengths.

The fully physical LPM makes this simple prediction of device performance, Eq. (5), based purely on specification of structural parameters, such as semiconductor layer compositions, widths and doping levels, and gate recess depth and width, without any prior experimental characterisation. The large-signal $\mathrm{RF}$ description makes a similar single subroutine call to the LPM, returning instantaneous drain-source voltage as a function of instantaneous values of source-gate voltage and source current. The main physical difference in the large-signal RF description is inclusion of a displacement contribution to the current, which provides a description of transistor capacitances.

\subsubsection{Low Computational Cost of the LPM}

The iterative solution of the electro-thermal equation, Eq. (4), requires repeated calls to the LPM via $\theta_{i}\left(P_{i}\right)$ for the active elements $i=1, \ldots, M$. Despite the speed of the quasi-2D LPM this can still be a significant computational expense. However, this computational cost can be reduced by noting that many of the calls to the LPM can occur for the same bias point, $\left(V_{D S}, V_{G S}\right)$, at similar values of the channel temperature, $T$. 
For illustration, assume the elements $i$ to be identical, e.g., a MMIC grid array for spatial power combining [54], with transistor fingers of identical layer structure and design, repeated between identical transistors on a MMIC and between identical MMICs in the array, so that $\theta_{i}\left(P_{i}\right)=\theta\left(P_{i}\right)$ for all $i$. Then for a given bias, the LPM can be called for a range of power dissipations, $P_{i}$, sufficient to span the range of $P_{i}$ generated in the iterative solution. By storing the resulting values $\theta\left(P_{i}\right)$ and making a simple interpolation between them, an essentially analytical description $\theta(P)$ can be generated for all $P$ of interest, at the cost of a small amount of memory storage for each bias point. This approach avoids wasteful repeated calls to the LPM in the vicinity of the same $\theta_{i}, P_{i}$ during iterative solution. These analytical $\theta(P)$ can be calculated prior to the fullycoupled electro-thermal solution. They will be generated rapidly, due to the speed of the LPM and provide the fully-coupled electro-thermal solution with all the physical predictive power and accuracy of the LPM, at the minimal computational cost of an essentially analytical function evaluation and small memory storage. The $\theta(P)$ will also provide essentially analytical derivatives for use in Newton-Raphson solution of the coupled non linear equation, Eq. (4).

\subsection{The Global Thermal Description}

As the electrical part of this coupled model is fully described by the existing LPM, the rest of the paper concentrates on the description, construction and demonstration of the global thermal model. Key considerations in the construction of the global thermal model were as follows:

1. It must provide rapid thermal updates in coupled electro-thermal solutions whilst providing a physical (hence predictive) and accurate description of steady-state heat flow.

2. It must require no core changes to the LPM.

3. It must treat the non linearity due to the temperature dependence of material parameters.
4. It must allow inclusion of radiative and convective surface fluxes in the description of large area systems.

5. It must allow treatment of totally arbitrary layouts and layer structures, providing a flexible tool for electro-thermal CAD.

To this end the analytical thermal resistance matrix approach was adopted. This approach is described by the key equation

$$
\Delta \theta_{i}=\sum_{j} R_{T H_{i j}} P_{j}
$$

which states that temperature rise, $\Delta \theta_{i}$, at active device element $i$, is determined entirely by the power dissipations $P_{j}$ at all active device elements, $j=1, \ldots, i, \ldots, M$, with matrix $\underline{R}_{T H}$ determined prior to the coupled electro-thermal calculation purely from structural information. The construction and demonstration of the consequences of such a relation, Eq. (7), for arbitrary MMIC and hybrid MIC geometries, form the subject of the rest of this paper. Key features of the analytical thermal resistance matrix approach are indicated below.

Firstly, as thermal updates in this description reduce to small matrix multiplications, the coupled electro-thermal solution is rapid. The thermal resistance matrix is obtained from analytical solutions of the heat diffusion equation with a linear 'radiation' boundary condition, so it treats both steady-state and time-dependent cases with surface radiation and convection. Non linearity due to temperature dependent thermal conductivity is treated by means of the Kirchhoff transformation [55]. Arbitrary 3-dimensional geometries are treated by interface matching of analytical solutions for thermal subelements (such as MMIC dies, air bridge legs and top, flip chips and solder bumps, and other surface metallisation).

Use of the $\underline{\underline{R}}_{T H}$ method means that no redundant temperature information is generated within the body of the device. Temperatures are only obtained at the active devices, as required for the coupled electro-thermal solution. (Though, as 
thermal solutions are analytical, once active device power dissipations have been obtained self-consistently, temperatures can be obtained at any point within the volume to any degree of accuracy. This is of value, for instance, in model validation by thermal imaging of surface temperatures.)

No surface or volume mesh is required in the analytical $\underline{\underline{R}}_{T H}$ method. Active elements and thermal subsystem interface discretisations are specified by the coordinates of the elementary area boundaries.

The thermal resistance matrix description $\underline{\underline{R}}_{T H}$ gives a one-to-one equivalent circuit model for the thermal interactions [17], Figure 4, which enables the immediate incorporation of the coupled electro-thermal description into general electrical network based solvers.

The form of the equivalent circuit illustrated in Figure $4[17,18]$ lends itself readily to extension to arbitrarily large numbers of nodes. This contrasts with some more conventional thermal equivalent circuit representations which attempt to identify directly circuit elements with obvious physical parameters, and which can grow rapidly more convoluted with increase in size [25]. The equivalent circuit description illustrated in Figure 4, and most immediately identified with the thermal resistance matrix formulation presented here, lends itself readily to circuit description of complex thermal problems.

\section{CONSTRUCTION OF THE RESISTANCE MATRIX}

Having presented the motivation for the analytical thermal resistance matrix approach, Eq. (7) is now derived for the thermal steady-state case and $\underline{\underline{R}}_{T H}$ obtained for the basic example of a homogeneous, heatsink mounted MMIC, bearing an arbitrary distribution of power transistor fingers.

This is followed by discussion of $\underline{\underline{R}}_{T H}$ for a MMIC comprised of multi-layers of differing thermal conductivity, which includes the cases of uniform surface metallisation, metal coated MMIC base, and doping dependent thermal conductivities in MMIC layers.

Treatment of MMIC superstructure is then presented followed by discussion of the construction of $\underline{\underline{R}}_{T H}$ using a double Fourier series finite element method for irregular surface metallisation. This is followed by construction of $\underline{\underline{R}}_{T H}$ for an inhomogeneous MMIC with vias or partial substrate thinning.

\subsection{Homogeneous MMIC}

The steady-state heat diffusion equation, in the absence of volume heat sources or sinks, is given by

$$
\nabla \cdot[\kappa(T) \nabla T]=0
$$

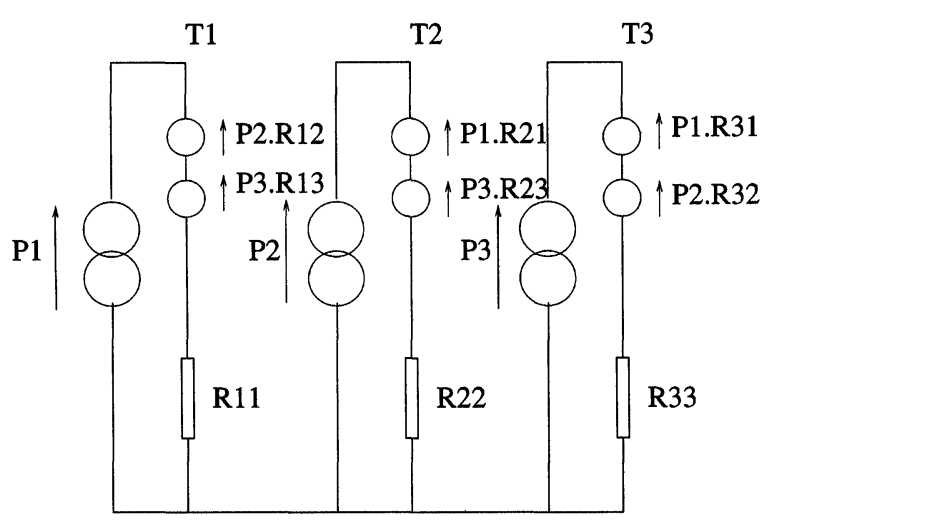

FIGURE 4 Thermal equivalent circuit corresponding to resistance matrix $\underline{\underline{R}}_{T H}[18]$. 
where $T$ is physical temperature and $\kappa(T)$ is temperature dependent thermal conductivity. This equation is non linear through $\kappa(T)$. The equation can be linearised by the Kirchhoff transformation [55]

$$
\theta=T_{S}+\frac{1}{\kappa_{S}} \int_{T_{S}}^{T} \kappa(T) d T,
$$

where $\kappa_{S}=\kappa\left(T_{S}\right)$. This gives the linearised steadystate heat diffusion equation

$$
\nabla^{2} \theta=0 .
$$

Then obtaining the analytical solution for linearised temperature $\theta$, physical temperature $T$ is immediately given by $T=T(\theta)$, where function $T(\theta)$ is determined from the Kirchhoff transformation, Eq. (9). For $\kappa(T)$ of the form

$$
\kappa(T)=\kappa_{S}\left(\frac{T}{T_{S}}\right)^{-b}
$$

appropriate to GaAs in temperature ranges of interest [56], $T(\theta)$ is given by [26]

$$
T(\theta)=T_{S}\left[1-\frac{(b-1)\left(\theta-T_{S}\right)}{T_{S}}\right]^{-1 /(b-1)} .
$$

To generate a solution of Eq. (10), the physical system must be specified and surface boundary conditions must be imposed. Equation (10) can be solved for general linear boundary conditions, but for simplicity adiabatic boundary conditions are assumed on the side faces of the MMIC. Active device power dissipation and heatsink mounting are described by a generalised 'radiation' boundary condition on the MMIC top and bottom surfaces. For illustration of the coordinate system, the structure of Figure 5 is considered. This consists of a GaAs MMIC, $0<x<L, 0<$ $y<W, 0<z<D$, bearing an arbitrary number of surface heating elements, areas $D_{i}$, dissipating (surface average) power densities, $P_{i}$ (corresponding to both active and passive devices).

Solving the linearised steady-state heat diffusion equation subject to adiabatic flux boundary conditions on the MMIC side faces, and the generalised linear radiation boundary condition,

$$
\alpha_{0, D} \kappa_{S} \frac{\partial \theta}{\partial z}+H_{0, D}\left[\theta-\theta_{0, D}(x, y)\right]+p_{0, D}(x, y)=0,
$$

on the MMIC top and bottom surfaces, the full solution for the linearised temperature by separation of variables is

$$
\begin{aligned}
\theta= & A+B z+\sum_{m n}^{\prime} \cos \lambda_{m} x \cos \mu_{n} y \\
& \times\left(C_{m n} \cosh \gamma_{m n} z+S_{m n} \sinh \gamma_{m n} z\right),
\end{aligned}
$$

where $\sum_{m n}^{\prime}$ means the sum over $m, n=0,1,2, \ldots$, excluding $(m, n)=(0,0)$,

$$
\lambda_{m}=\frac{m \pi}{L},
$$

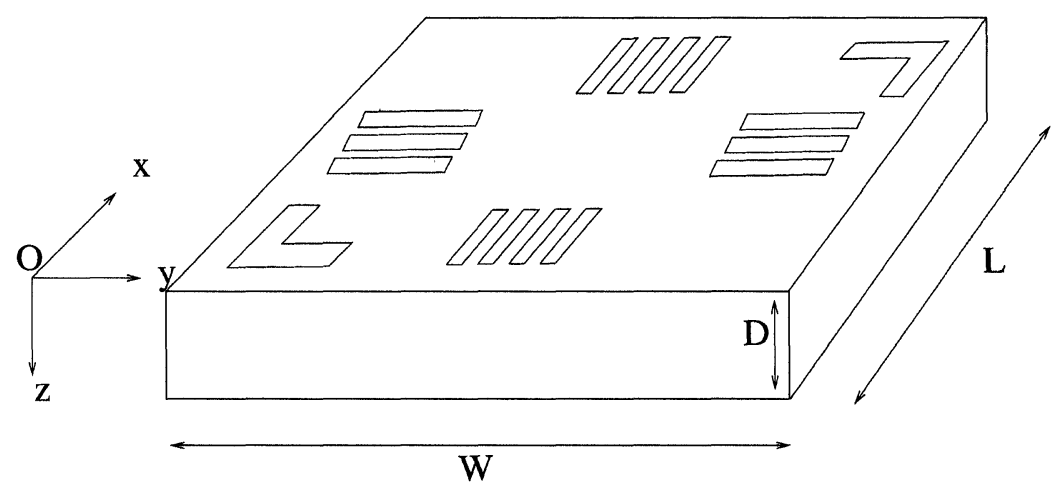

FIGURE 5 Illustrative GaAs MMIC for generation of $R_{T H}$. 


$$
\begin{gathered}
\mu_{n}=\frac{n \pi}{W} \\
\gamma_{m n}^{2}=\lambda_{m}^{2}+\mu_{n}^{2} \\
H_{D} A=-\frac{1}{L W} \int_{0}^{L} \int_{0}^{W} p_{D}(x, y)-H_{D} \theta_{D}(x, y) d x d y \\
-\left(\alpha_{D} \kappa_{S}+H_{D} D\right) B \\
\kappa_{S} \alpha_{0} B=-\frac{1}{L W} \int_{0}^{L} \int_{0}^{W} p_{0}(x, y) \\
-H_{0} \theta_{0}(x, y) d x d y-H_{0} A \\
H_{0} C_{m n} \\
=-\gamma_{m n} S_{m n} \alpha_{0} \kappa_{S} \\
-\frac{\int_{0}^{L} \int_{0}^{W} \cos \lambda_{m} x \cos \mu_{n} y\left[p_{0}(x, y)-H_{0} \theta_{0}(x, y)\right] d x d y}{(L W / 4)\left(1+\delta_{m 0}\right)\left(1+\delta_{n 0}\right)}
\end{gathered}
$$

and

$$
\begin{aligned}
& C_{m n}\left[\alpha_{D} \kappa_{S} \gamma_{m n} \sinh \gamma_{m n} D+H_{D} \cosh \gamma_{m n} D\right] \\
& +S_{m n}\left[\alpha_{D} \kappa_{S} \gamma_{m n} \cosh \gamma_{m n} D+H_{D} \sinh \gamma_{m n} D\right] \\
& =-\frac{\int_{0}^{L} \int_{0}^{W} \cos \lambda_{m} x \cos \mu_{n} y\left[p_{D}(x, y)-H_{D} \theta_{D}(x, y)\right] d x d y}{(L W / 4)\left(1+\delta_{m 0}\right)\left(1+\delta_{n 0}\right)}
\end{aligned}
$$

Choosing $\quad \alpha_{0}=1, \quad \alpha_{D}=0, \quad H_{0}=0, \quad H_{D}=1$, $p_{0}(x, y)=\sum_{i} S_{i}(x, y) P_{i}, \quad p_{D}(x, y)=0, \quad \theta_{0}(x, y)=0$, $\theta_{D}(x, y)=T_{S}$ describes a homogeneous, heatsink mounted MMIC at base temperature $T_{S}$ with imposed surface flux $p_{0}(x, y)$ due to active device elements, and no surface convective or radiative losses. Here $S_{i}(x, y)$ equals 1 in elementary areas $D_{i}$ dissipating uniform power densities $P_{i}$, and 0 otherwise.

This gives the explicit form for the linearised temperature throughout the MMIC,

$$
\begin{aligned}
\theta= & T_{S}-\frac{z-D}{\kappa_{S} L W} \sum_{i} I_{00}^{i} P_{i} \\
& +\sum_{m n}^{\prime} \cos \lambda_{m} x \cos \mu_{n} y \\
& \times\left(\sinh \gamma_{m n} z-\tanh \gamma_{m n} D \cosh \gamma_{m n} z\right) \\
& \times \frac{-4}{\kappa_{S} L W\left(1+\delta_{m 0}\right)\left(1+\delta_{n 0}\right) \gamma_{m n}} \sum_{i} I_{m n}^{i} P_{i},
\end{aligned}
$$

where elementary area integral $I_{m n}^{i}$ is defined by

$$
I_{m n}^{i}=\iint_{D_{i}} \cos \lambda_{m} x \cos \mu_{n} y d x d y
$$

and the standard result,

$$
\int_{0}^{L} \cos \lambda_{m} x \cos \lambda_{m^{\prime}} x d x=\frac{L}{2} \delta_{m m^{\prime}}\left(1+\delta_{m 0} \delta_{m^{\prime} 0}\right)
$$

has been used (and similarly for the corresponding $y$ integral) with $\delta_{m n}$ the Kronecker delta function.

To construct the thermal resistance matrix, surface averaged temperature rises, $\Delta \theta_{i}$, are evaluated as

$$
\Delta \theta_{i}=\frac{\iint_{D_{i}} \theta-T_{S} d x d y}{\iint_{D_{i}} d x d y} .
$$

Then Eq. (7) is obtained immediately with

$$
\begin{aligned}
& R_{T H_{i j}} \\
& =\frac{1}{\kappa_{S} L W}\left[D I_{00}^{j}+\sum_{m n}^{\prime} \frac{4 \tanh \gamma_{m n} D}{\gamma_{m n}\left(1+\delta_{m 0}\right)\left(1+\delta_{n 0}\right)} \frac{I_{m n}^{i} I_{m n}^{j}}{I_{00}^{i}}\right] .
\end{aligned}
$$

This expression illustrates the main points of the analytical thermal resistance matrix approach. The thermal resistance matrix is evaluated just once, prior to coupled electro-thermal calculation, purely in terms of structural parameters. As it is obtained for linearised temperature, it is independent of both temperature and power, and hence independent of electrical bias point. With the non linear relation

$$
\Delta \theta_{i}=\Delta \theta_{i}\left(P_{i}\right),
$$

from the physical active device model, combination with the global thermal description, Eq. (7), gives

$$
\Delta \theta_{i}\left(P_{i}\right)=\sum_{j} R_{T H_{i j}} P_{j}
$$

which is a small, simple, non linear system to be solved self-consistently for the power densities, $P_{j}$. 
Having obtained the $P_{j}$ from solution of the coupled electro-thermal problem, for instance by Newton methods, the full electrical solution is obtained. Also, the temperature at any point within the MMIC is then given, if required, by Eq. (22).

As an illustration, the surface temperature distribution calculated using the coupled electrothermal HEMT model is shown in Figure 6 for a power HEMT with 60 fingers. Total power dissipated is $2.8 \mathrm{~W}, T_{S}=300 \mathrm{~K}$, and plot temperature varies from below $36^{\circ} \mathrm{C}$ to $53^{\circ} \mathrm{C}$. Calculation of a full set of $\mathrm{I}-\mathrm{V}$ curves for this device took some 10 's of minutes based on a non optimised relaxation algorithm.

The simple expression, Eq. (26), represents a full 3-dimensional solution of the heat diffusion equation for a MMIC with an arbitrary surface distribution of power transistors (and heat dissipating passive elements), each power transistor having an arbitrary grouping and arrangement of transistor fingers. It includes fully, finite length and end effects for heat diffusion from the active layers, and treats the finite volume effects of the die without any need for the simplifying assumption of an infinite or semi-infinite substrate. The extension to other realisations of the radiation boundary condition, Eq. (13), for instance deriving simple resistance matrix expressions for large area substrates with convective and radiative surface losses, is immediate.

Use of the expression, Eq. (26), in coupled electro-thermal simulations of power FETs and MMICs, neglects the effects of surface metallisa- tion and resulting heat spreading. It will therefore generally overestimate the magnitude of $\underline{\underline{R}}_{T H}$, producing simulated device temperatures higher than those realised experimentally. Simple use of Eq. (26) can, therefore, only to be expected to produce a safe upper limit on temperature rises. It will give correct order of magnitude estimates for temperature rises, and demonstrate correct trends in temperature variation with changes in parameters, but accurate prediction of $\underline{\underline{R}}_{T H}$ based purely on the physical thermal model will typically require description of metallic superstructure, as described in the sections following.

\subsection{Generalisation of $\underline{\underline{R}} T H$}

In Eq. (25) of the previous section, surface average temperature rises were calculated for the elementary areas, $D_{i}$, over which the active device power densities, $P_{i}$, were dissipated. As temperature rises in a multi-finger device will typically be highest in the immediate vicinities of peak power dissipations, the thermal resistance matrix of Eq. (26) will return peak temperatures.

For use in the coupled electro-thermal formulation, with the LPM employing a uniform channel temperature approximation, the active device temperature that is fed into the electrical model should be the average temperature over the whole source-gate-drain area. This means that the construction of surface average temperature rises, as in Eq. (25), should be modified so that temperatures are averaged over source-gate-drain areas,

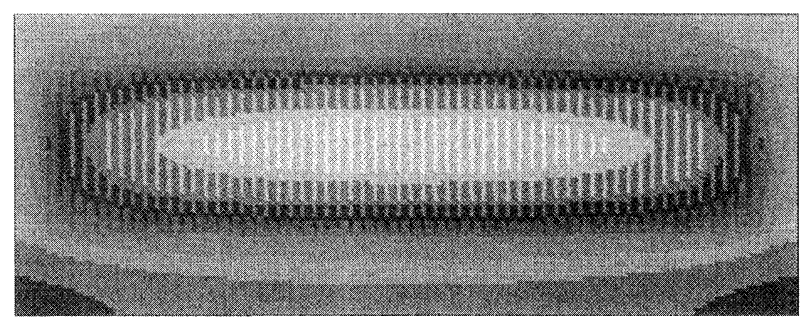

FIGURE 6 Coupled electro-thermal calculation of surface temperature distribution for a 60 -finger power HEMT. 
$D_{i}^{\prime}$, corresponding to each active device power dissipating area, $D_{i}$.

This gives immediately for the generalised thermal resistance matrix,

$$
\begin{aligned}
& R_{T H_{i j}} \\
& =\frac{1}{\kappa_{S} L W}\left[D I_{00}^{j}+\sum_{m n}^{\prime} \frac{4 \tanh \gamma_{m n} D}{\gamma_{m n}\left(1+\delta_{m 0}\right)\left(1+\delta_{n 0}\right)} \frac{I_{m n}^{\prime i} I_{m n}^{j}}{I_{00}^{i}}\right],
\end{aligned}
$$

where elementary area integrals $I_{m n}^{\prime i}, I_{00}^{\prime i}$ are still of the form, Eq. (23), but now integrations are over areas $D_{i}^{\prime}$.

More generally, having obtained an analytical solution of the form, Eq. (22), temperature rises can be calculated at any point, or averaged over any area or volume, irrespective of any correlation to power dissipating areas. Thermal resistance matrices so constructed will generally be of a different explicit form to Eqs. (26) and (29) and will not generally be square.

Use of source-gate-drain areas, $D_{i}^{\prime}$, in the thermal resistance matrix, is to be distinguished from use of an effective 'hot strip' width much wider than the dimensions of the physical power dissipating area, as in [16]. In [16], a wide active power dissipating area was assumed to obtain agreement with low resolution thermal measurements. In the formulation presented here, the extents of the power dissipating regions, $D_{i}$, are physical and are obtained from the LPM. The use of source-gate-drain averaged temperature rises over areas, $D_{i}^{\prime}$, is required for physical consistency with the uniform channel temperature version of the LPM. The effects of low resolution in thermal measurements can be simulated by averaging the analytical result, Eq. (22), over pixel sized areas, after generation of the self-consistent electrothermal solution.

\subsection{Multilayer MMIC}

The simple description of the homogeneous MMIC, presented above, is readily generalised to treat multi-layer systems by use of a transfer matrix, or two-port network, approach [9]. This is based on matching of Fourier components at interfaces, and corresponds to use of the double cosine transform to convert the 3-dimensional partial differential equation, Eq. (10), into a 1dimensional ordinary differential equation for the $z$-dependent double Fourier series coefficients. Matching of linearised temperature and flux at the interfaces of a multi-layer structure can then be imposed by use of a $2 \times 2$ transfer matrix on the Fourier series coefficients and their derivatives. Arbitrary N-level structures can be treated. Different thermal conductivities can be assumed in each layer allowing treatment of composites like $\mathrm{Cu}$ on $\mathrm{AlN}$ (both having temperature independent thermal conductivities) and MMICs with conductivities varying from layer to layer due to differences in doping levels (all layers having the same functional form for the temperature dependence of the conductivity). The method can be generalised further, by imposing specified flux discontinuities at the interfaces. The solution then represents, for instance, the case of a MMIC with active device channel buried by a thin layer of semiconductor.

For instance, consider a 2-layer structure of the general form of Figure 5, but with top layer $0<z<D_{1}$, thermal conductivity $\kappa_{1}$ and bottom layer $D_{1}<z<D_{2}$, thermal conductivity $\kappa_{2}$. With power dissipations $P_{i}$ over elementary areas $D_{i}$ at the interface, $z=D_{1}$, the (linearised) temperature distributions $\theta^{(1)}$ and $\theta^{(2)}$, in the top and bottom layers respectively, are given by

$$
\theta^{(1)}=A^{(1)}+\sum_{m n}^{\prime} \cos \lambda_{m} x \cos \mu_{n} y C_{m n}^{(1)} \cosh \gamma_{m n} z
$$

$$
\begin{aligned}
\theta^{(2)}= & T_{S}+B^{(2)}\left[z-\left(D_{1}+D_{2}\right)\right] \\
& +\sum_{m n}^{\prime} \cos \lambda_{m} x \cos \mu_{n} y S_{m n}^{(2)} \\
& \times\left[\sinh \gamma_{m n} z-\tanh \gamma_{m n}\left(D_{1}+D_{2}\right) \cosh \gamma_{m n} z\right],
\end{aligned}
$$


where,

$$
\begin{gathered}
A^{(1)}=T_{S}-B^{(2)} D_{2}, \\
C_{m n}^{(1)}=S_{m n}^{(2)}\left[\tanh \gamma_{m n} D_{1}-\tanh \gamma_{m n}\left(D_{1}+D_{2}\right)\right] \\
B^{(2)}=\frac{-1}{\kappa_{2} L W} \sum_{i} I_{00}^{i} P_{i},
\end{gathered}
$$

and

$$
\begin{aligned}
S_{m n}^{(2)}= & \frac{4 \operatorname{cosech} \gamma_{m n} D_{1}}{L W\left(1+\delta_{m 0}\right)\left(1+\delta_{n 0}\right) \gamma_{m n}} \sum_{i} I_{m n}^{i} P_{i} \\
& \times \frac{1}{\left\{\begin{array}{c}
\kappa_{1}\left[\tanh \gamma_{m n} D_{1}-\tanh \gamma_{m n}\left(D_{1}+D_{2}\right)\right] \\
-\kappa_{2}\left[\operatorname{coth} \gamma_{m n} D_{1}-\tanh \gamma_{m n}\left(D_{1}+D_{2}\right)\right]
\end{array}\right\}} .
\end{aligned}
$$

The expression for the corresponding thermal resistance matrix, describing the temperature rises of the power dissipating elements at the interface, is

$$
\begin{aligned}
R_{T H_{i j}}=\frac{1}{\kappa_{2} L W}[ & D_{2} I_{00}^{j}+\sum_{m n}^{\prime} \frac{4}{\left(1+\delta_{m 0}\right)\left(1+\delta_{n 0}\right) \gamma_{m n}} \frac{I_{m n}^{i} I_{m n}^{j}}{I_{00}^{i}} \\
& \times\left(\tanh \gamma_{m n}\left(D_{1}+D_{2}\right)-\tanh \gamma_{m n} D_{1}\right) / \\
& \left(1-\frac{\kappa_{1}}{\kappa_{2}} \tanh ^{2} \gamma_{m n} D_{1}-\left(1-\frac{\kappa_{1}}{\kappa_{2}}\right)\right. \\
& \left.\left.\times \tanh \gamma_{m n}\left(D_{1}+D_{2}\right) \tanh \gamma_{m n} D_{1}\right)\right] .
\end{aligned}
$$

Adopting an effective value for $\kappa_{1}$, to allow for the different functional forms of temperature dependent thermal conductivity in metal and GaAs, this expression can be used to provide a simple approximation to the thermal resistance matrix of a heavily metallised power FET or MMIC.

\subsection{MMIC Superstructure}

It has been demonstrated that inclusion of surface metallisation is essential for accurate description of thermal effects in power devices $[4,5,7]$.
Comparison with experiment for multi-finger power HBTs shows that the simple thermal description corresponding to the resistance matrix of Eq. (26) is highly accurate when combined with a simple model of heat shunting by an air bridge [17]. The extension of the analytical thermal resistance matrix approach to include descriptions of surface metallisation, air bridges and other vertical geometries such as flip chips and solder bumps, is now presented.

The extension to include surface superstructure is achieved by solving the heat diffusion equation analytically for thermal sub elements, then combining thermal resistance matrices for subsystems by matching of temperature and flux at discretised interfaces. In the case discussed below, the multilayer problem is solved for discretised temperatures at thermal sub element interfaces. However, the matching problem can equally be cast in terms of solution for discretised interface fluxes. The former method is described here, as the latter produces a more complicated formulation for the system of equations to be solved for the interface unknowns. (This is because specification of flux boundary conditions on top and bottom surfaces of a thermal sub element only defines temperature upto an arbitrary constant, and imposes a subsidiary energy conservation constraint on total flux into and out of the volume. When matching sub elements at interfaces, these energy conservation constraints serve to determine the arbitrary constants in the temperatures, but require moderately complicated manipulation of the simultaneous equations for the interface unknowns, to cast them in terms of only independent fluxes and the related arbitrary temperature constants.)

To generate analytical solutions allowing interface matching of thermal sub elements, $\alpha_{0}, \alpha_{D}, H_{0}$, $H_{D}, p_{0}(x, y), p_{D}(x, y), \theta_{0}(x, y)$ and $\theta_{D}(x, y)$ of Eq. (13) are specified as in Section 4.1, but with $p_{0}(x, y)$ written $p_{0}(x, y)=\sum_{i} S_{0 i}(x, y) P_{0 i}$ and the uniform MMIC base temperature replaced by the discretised temperature distribution $\theta_{D}(x, y)=\sum_{j} S_{D j}$ $(x, y) \theta_{D j}$. Similarly for thermal sub element surface temperature, $\theta_{0 i}$, and base flux, $P_{D j}$. Then 
following the procedure of Section 4.1, specifying either discretised base temperature or base flux, the following relations are obtained:

$$
\begin{aligned}
& \theta_{0 i}=\sum_{i^{\prime}} R_{T H_{i i^{\prime}}} P_{0 i^{\prime}}+\sum_{j} Z_{i j} \theta_{D j}, \\
& P_{D j}=\sum_{i} S_{T H_{j i}} P_{0 i}+\sum_{j^{\prime}} T_{j j^{\prime}} \theta_{D j^{\prime}},
\end{aligned}
$$

where $R_{T H_{i t^{\prime}}}$ is given by Eq. (26) and

$$
\begin{aligned}
& Z_{i j}= \frac{1}{L W}\left[I_{00}^{D j}+\sum_{m n}^{\prime} \frac{4 \operatorname{sech} \gamma_{m n} D}{\left(1+\delta_{m 0}\right)\left(1+\delta_{n 0}\right)} \frac{I_{m n}^{0 i} I_{m n}^{D j}}{I_{00}^{0 i}}\right], \\
& S_{T H_{j i}}= \frac{1}{L W}\left[I_{00}^{0 i}+\sum_{m n}^{\prime} \frac{4 \operatorname{sech} \gamma_{m n} D}{\left(1+\delta_{m 0}\right)\left(1+\delta_{n 0}\right)} \frac{I_{m n}^{D j} I_{m n}^{0 i}}{I_{00}^{D j}}\right] \\
& T_{j j^{\prime}}=-\alpha \frac{1}{L W} \sum_{m n}^{\prime} \frac{4}{\left(1+\delta_{m 0}\right)\left(1+\delta_{n 0}\right)} \frac{I_{m n}^{D j}}{I_{00}^{D j}} \\
& \times \sum_{k=1}^{N=1}\left(I_{m n}^{D k}-\frac{I_{m n}^{D N} I_{00}^{D k}}{I_{00}^{D N}}\right)\left(\underline{\underline{R}}_{D D}^{-1}\right)_{k j^{\prime}},
\end{aligned}
$$

with

$$
\begin{aligned}
& R_{D D_{j j^{\prime}}=} \\
& \begin{aligned}
\delta_{N j^{\prime}}+\alpha\left(1-\delta_{N j^{\prime}}\right) \frac{1}{\kappa_{S} L W} \sum_{m n}^{\prime} & \frac{4 \operatorname{coth} \gamma_{m n} D}{\left(1+\delta_{m 0}\right)\left(1+\delta_{n 0}\right.} \frac{1}{\left.\gamma_{m n}\right)} \\
& \times \frac{I_{m n}^{D j}}{I_{00}^{D j}}\left(I_{m n}^{D j^{\prime}}-\frac{I_{m n}^{D N} I_{00}^{D j^{\prime}}}{I_{00}^{D N}}\right) .
\end{aligned}
\end{aligned}
$$

$I_{m n}^{0 i}$ and $I_{m n}^{D j}$ are the area integrals of Eq. (23) evaluated over elementary areas $D_{i}$ on the surface $z=0$, and over elementary areas $D_{j}$ on the base, $z=D$, respectively. $N$ is the number of discretised base elementary areas, $D_{j}$, and $\alpha$ takes any value with dimensions $\mathrm{W} /\left(\mathrm{K} \cdot \mathrm{m}^{2}\right)$.

To illustrate the interface matching approach, the global thermal resistance matrix is now constructed for the case of $\mathrm{N}$ pieces of rectangular, but otherwise arbitrary, metallisation on the surface of an otherwise homogeneous heatsink mounted MMIC. For simplicity of illustration, only a single metal layer is considered (though each individual piece of surface metal can be of any required thickness). The generalisation of the this approach to multilayer structures such as air bridges and flip chips is immediate.

For a single metal layer, surface flux terms, $P_{0 i}$, in Eqs. (37) and (38) are identically zero. As the surface temperature of the metal, $\theta_{0 i}$, is not required for the coupled electro-thermal solution, only Eq. (38) is required. Written in matrix notation this becomes simply

$$
\underline{P}_{D}^{(n)}=\underline{\underline{T}}^{(n)} \underline{\theta}_{D}^{(n)}
$$

for each piece of surface metallisation, $n$, with $n=1, \ldots, N$. The thermal conductivity $\kappa_{S}$ in $\underline{\underline{T}}^{(n)}$, Eq. (39), for each piece of metallisation, $n$, can be chosen at a different representative Kirchhoff transformation temperature, $T_{S}$. Such a choice allows more accurate treatment of the difference in functional form between conductivity in GaAs and metal [57]. This technique is described in more detail in Section 4.5 for the inhomogeneous MMIC with vias.

In matrix notation, Eq. (7) for the MMIC die becomes

$$
\left(\begin{array}{l}
\underline{\theta}^{s}-\underline{T}_{S}^{s} \\
\underline{\underline{i}}^{i}-\underline{T}_{S}^{i}
\end{array}\right)=\left(\begin{array}{ll}
\underline{R}_{T H}^{s s} & \underline{R^{s i}} \\
\underline{R}_{T H}^{i s} & \underline{\underline{R}}
\end{array}\right.
$$

where $T_{S}{ }^{s}$ and $\underline{T}_{S}^{i}$ are constant vectors with all elements equal to $T_{S}$, and the matrix equation has been partitioned by active device elementary surface areas, $s$, and interface elementary areas between MMIC die and metal, $i$.

Matching flux and (linearised) temperature at the interface between metal and MMIC die, the following relation is obtained

$$
\underline{\Delta \theta}^{s}=\underline{\underline{R}}_{T H}^{\mathrm{glob}} \underline{P}^{s},
$$

where $\underline{P}^{s}$ is the vector of MMIC active device power dissipations, $\underline{\underline{R}}_{T H}^{\text {glob }}$ is the global thermal resistance matrix for the coupled GaAs and metal system, and $\underline{\Delta \theta}$ is the vector of MMIC active device temperature rises. 
The global resistance matrix is given explicitly by

$$
\underline{\underline{R}}_{T H}^{\mathrm{glob}}=\underline{\underline{R}}_{T H}^{s s}+\underline{\underline{R}}_{T H}^{s i} \underline{\underline{R}} \underline{\underline{R}}_{T H}^{i s},
$$

where

$$
\begin{aligned}
\underline{\underline{R}}= & \operatorname{diag}\left(\underline{\underline{T}}^{(1)}, \ldots, \underline{\underline{T}}^{(n)}, \ldots, \underline{\underline{T}}^{(N)}\right) \\
& \times\left[\underline{\underline{I}}^{i}-\underline{\underline{R}}_{T H}^{i i} \operatorname{diag}\left(\underline{\underline{T}}^{(1)}, \ldots, \underline{\underline{T}}^{(n)}, \ldots, \underline{\underline{T}}^{(N)}\right)\right]^{-1},
\end{aligned}
$$

$\underline{I}^{i}$ is the identity matrix, and temperature rises $\underline{\underline{\Delta}}{ }^{s}$ are now defined by

$$
\underline{\Delta \theta^{s}}=\underline{\theta}^{s}-\left(\underline{\underline{I}}^{s}+\underline{\underline{R}}_{T H}^{s i} \underline{\underline{R}}\right){\underline{T_{S}}}^{s},
$$

where, again, $\underline{\underline{I}}^{s}$ is the identity matrix.

Equation (42) illustrates the main points of the hierarchical construction of global thermal resistance matrices for complex systems. Firstly, the order of the global thermal resistance matrix, $\underline{R}_{T H}^{\text {glob }}$, is small, determined only by the number of active device elements, not the level of internal discretisation. This implies rapid temperature updates in the iterative coupled electro-thermal solution. Secondly, $\underline{\underline{R}}_{T H}^{\text {glob }}$ is constructed purely from simple matrix manipulations on elementary matrices given by simple analytical expressions for thermal subsystems. The global resistance matrix is constructed just once, prior to the coupled electro-thermal solution, so its construction has no impact on coupled run time. The only significant approximation in construction of $\underline{R}_{T H}^{\text {glob }}$ is the level of interface discretisation. This is determined only by available computing power and required accuracy, and description by $\underline{\underline{R}}_{T H}^{\text {glob }}$ becomes exact in the limit of infinitely fine interface discretisation. Bonani et al., have typically found $1000-2000$ nodes to be sufficient in their hybrid finite element Green's function description of MMIC surface metallisation [2,3].

\subsubsection{Non Linear Interface Matching}

In the hierarchical treatment of MMIC superstructure just presented, linearised temperatures were matched at subsystem interfaces. This is only strictly justified when the functional form of the temperature dependence of the thermal conductivity is the same in both matched layers. When the functional forms are different, matching of physical temperature, $T$, imposes a non linear relation on the linearised temperatures, $\theta$, in the two subsystems. This arises as a result of the different relations $T=T(\theta)$ obtained from the respective Kirchhoff transformations, Eqs. (9), (11) and (12). For GaAs, exponent $b$ in Eq. (11) is typically equal to 1.22 [56] whereas for metal $b$ equals 0 , i.e., the thermal conductivity of metal is essentially temperature independent. This difference means that matching of linearised temperatures at the GaAs/metal interfaces implies a non linear matching relation. (Matching of fluxes, however, remains linear after differing Kirchhoff transformations.)

This non linear interface matching can be avoided to a good approximation, by assuming the same functional form for the temperature dependent thermal conductivity of the metal and of the GaAs. The resulting error in surface temperatures is calculated to be typically a few per cent $[7,57]$. This error can be reduced further in multi-component metal on GaAs systems, by assuming a different Kirchhoff transformation temperature, $T_{S}$, for each piece of surface metallisation. Choosing these Kirchhoff transformation temperatures to give a thermal conductivity $\kappa_{S}$ close to the metal thermal conductivity, minimises the errors introduced by adoption of an incorrect functional form for the temperature dependence.

In cases where the non linear interface matching cannot be neglected, the thermal resistance matrix approach allows formulation of a non linear system of equations for the correctly matched temperatures [19]. For the case of a grid array of identical GaAs MMICs on an AIN substrate (with essentially temperature independent thermal conductivity), arguments similar to those of Section 4.4 give rise to the non linear system for interface temperatures $\underline{\theta}_{D}^{(n)}$ of MMICs 
$n=1, \ldots, M$

$$
N L\left(\underline{\theta}_{D}^{(n)}\right)=\sum_{m} \underline{R}_{T H_{n m}}^{\mathrm{sub}}\left(\underline{S}_{T H} \underline{P}_{0}^{(m)}+\underline{T} \underline{\theta}_{D}^{(m)}\right),
$$

in terms of the MMIC surface flux densities $\underline{P}_{0}^{(m)}$. $N L(\theta)$ represents the non linear relation between temperatures matched at the interfaces. The substrate thermal resistance matrix $\underline{R}_{T H}^{\text {sub }}$ has been partitioned into submatrices $\underline{R}_{T H_{n m}}^{\text {sub }}$, with $n, m=$ $1, \ldots, M$ running over the individual MMICs, and $\underline{S}_{T H}$ and $\underline{\underline{T}}$ are defined as in Eq. (39). Solving self-consistently for the interface temperatures, Eq. (37) provides a non linear relation between surface temperatures $\underline{\theta}_{0}^{(n)}$ and surfaces fluxes $\underline{P}_{0}^{(m)}$ for the MMIC grid array.

This non linear problem for the interface temperatures can again be solved by Newton methods. The number of unknowns is determined by the level of discretisation of the MMIC bases, and can be minimised by choosing the shapes of the elementary areas to reflect the expected behaviour of the MMIC base temperature profiles.

Figures 7 and 8 illustrate the simulation of a $5 \times 5$ power FET array, with explicit non linear matching of $\mathrm{MMIC} /$ substrate interface temperatures. The MMIC array substrate was heatsink mounted and adiabatic surface boundary conditions were assumed. Single surface averaged MMIC base temperatures were matched at the MMIC-substrate interfaces. The coupled electro-thermal simulation assumed non linear matching of linearised temperatures at the interfaces, due to the different functional forms of the temperature dependences in GaAs MMICs and AIN substrate. Coupled electro-thermal simulation time for 1 DC bias point was $\sim 10$ minutes (after construction of the respective thermal resistance matrices). This was based on a simple non optimised relaxation algorithm and repeated calls to the LPM in the iterative coupled solution. Use of Newton methods, combined with prior calculation and interpolation for the LPM temperature dependence, is expected to produce significant further speed-up up in simulation run time which was dominated by calls to the LPM.

\subsubsection{Non Linear Surface Fluxes}

Surface radiative fluxes are unimportant for the small areas of heatsink mounted MMICs and even

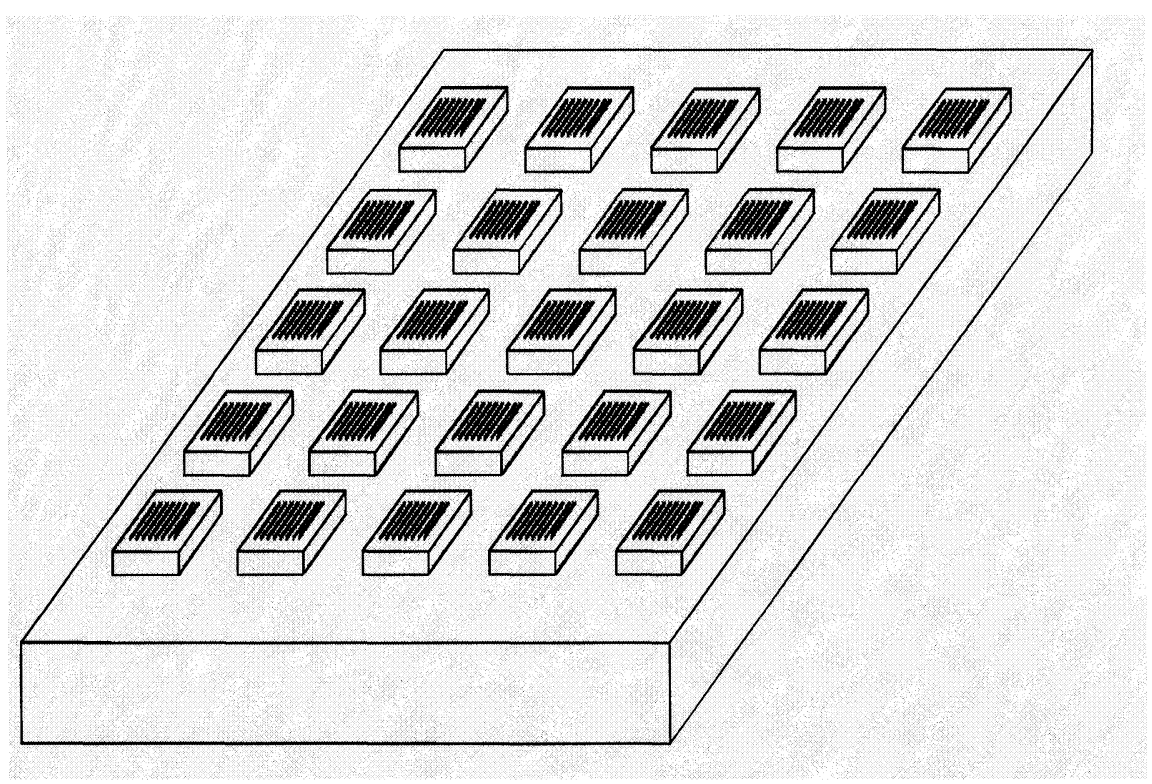

FIGURE 7 Illustration of $5 \times 5$ power FET array simulated electro-thermally. 


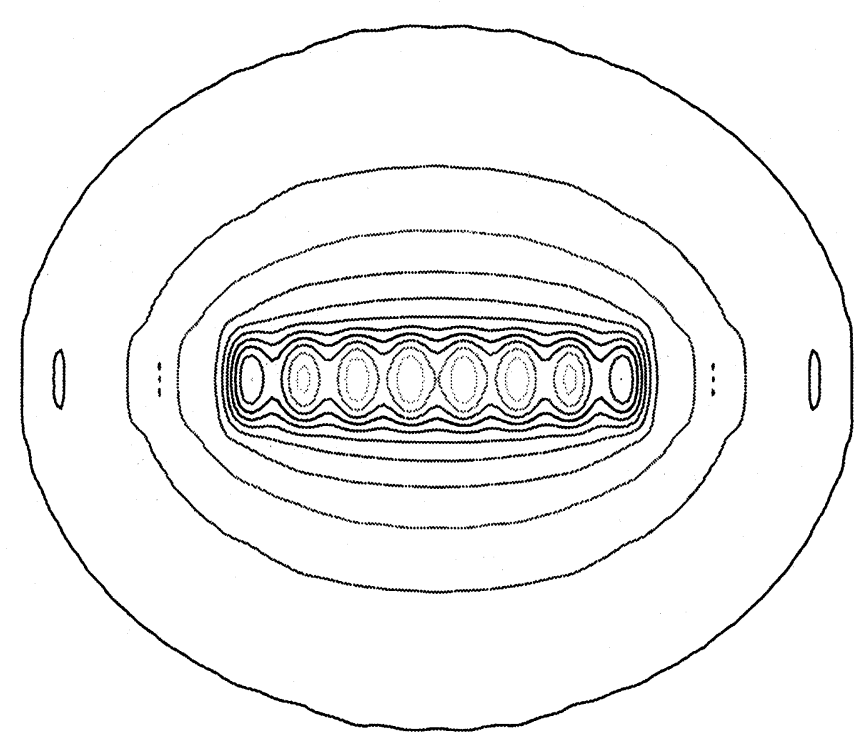

FIGURE 8 Electro-thermal simulation of the central power FET of a $5 \times 5$ power FET array. Non linear temperature matching was used at MMIC-substrate interfaces.

hybrid MICs. Convective fluxes are non trivial to calculate for small areas and fine surface structure. However, for large area substrates, from MMIC grid arrays upto circuit board level, convective and radiative losses are known to become significant and at high powers and temperatures surface flux non linearities need to be considered. The linear thermal resistance matrix approach allows treatment of non linear surface fluxes by discretising the whole substrate surface (not just the active device elements) and treating the radiative and convective surface fluxes as unknown imposed fluxes to be obtained self-consistently.

Adopting this approach, Eq. (7) gives immediately the following non linear relation for the surface temperature rises $\Delta \theta_{i}[19]$,

$$
\begin{aligned}
\Delta \theta_{i}= & \sum_{j} R_{T H_{i j}} P_{j}-\sum_{r} Q_{T H_{i r}} n l\left(\theta_{r}\right) \\
& -\sum_{s} V_{T H_{i s}} n l\left(\theta_{s}^{\mathrm{rev}}\right)
\end{aligned}
$$

in terms of the imposed surface fluxes $P_{j} \cdot n l(\theta)$ is the surface flux non linearity, $\theta_{r}$ the substrate surface temperature on the front surface, and $\theta_{s}^{\text {rev }}$ substrate temperature on the reverse surface. $Q_{T H_{i r}}$ and $V_{T H_{i s}}$ are thermal resistance matrices.

This relation leads immediately to an iterative solution for treatment of surface flux non linearity. As the non linear contribution to the surface flux is obtained as a perturbation about the linear radiation boundary condition, and as the linear case can be solved to provide a good first guess in the iterative non linear solution, the problem is expected to be rapidly convergent and stable.

The required level of surface discretisation and hence the number of unknowns, $\Delta \theta_{i}$, in the non linear system, will depend on the magnitude of the substrate thermal conductivity and the corresponding level of uniformity of the substrate temperature.

Where the non linearity is too strong to allow rapid solution via the iteration of the linear thermal resistance matrix, direct solution of the heat diffusion equation for arbitrary non linear boundary conditions can be achieved by a combination of the double Fourier series expansion solution, Eq. (14), and a collocation technique. This solution is exact to the extent that the double Fourier series has converged. This 
approach produces a non linear thermal resistance function $R_{T H}\left(P_{1}, \ldots, P_{i}, \ldots, P_{N}\right)$. The solution is computationally much more expensive than simple linear matrix construction, but possibly of value for high accuracy at high power dissipations and temperatures. The linear thermal resistance matrix solution requires surface discretisation and corresponding thermal terminals in a thermal network solution for non linear surface fluxes. By contrast, the non linear thermal resistance function provides a description of arbitrary surface flux non linearity without introducing additional surface terminals in the non linear thermal network.

\subsection{3. $\underline{\underline{R}}_{T H}$ from Finite Element or Finite Difference Schemes}

The generation of the thermal resistance matrix has so far been described on the basis of analytical solutions of the heat diffusion equation. However, $\underline{\underline{R}}_{T H}$ can also be generated experimentally or by numerical simulation. Direct construction of $\underline{\underline{R}}_{T H}$ based on physical temperatures obtained from non linear experiment or simulation, only allows linearisation in a small signal sense, and is of very limited applicability. However, if the system non linearity can be attributed almost totally to temperature dependent conductivity, i.e., if surface flux non linearities are negligible, then physical temperatures can be converted to linearised temperatures via the Kirchhoff transformation, Eq. (9), and a thermal resistance matrix for linearised temperature can be constructed. The thermal description so obtained is independent of temperature and power dissipation, and so of device bias point. It is not restricted to small temperature variations.

Numerical simulations offer an increased degree of flexibility beyond that obtainable by experiment. Where a system is composed of subsystems with different functional forms for the thermal conductivity, each subsystem can be simulated separately, and a thermal resistance matrix constructed for linearised temperature in each case. Solving a system of non linear equations for matched interface temperatures again gives a thermal description valid for all temperatures and power densities and is not limited to small temperature swings.

To generate $\underline{\underline{R}}_{T H}$ experimentally or numerically, Eq. (7) gives the definition

$$
R_{T H_{i j}}=\left.\frac{\Delta \theta_{i}}{P_{j}}\right|_{P_{r}=0, r \neq j} .
$$

Generation of a thermal resistance matrix of order $N \times N$, corresponding to $N$ active device elements, therefore requires $N$ individual measurements or simulations, dissipating power in just one active device at a time, and measuring the resulting temperature rises at all active devices. (The number of measurements can be reduced if the system is symmetrical.) However, to generate experimentally or numerically, a thermal resistance matrix giving comparable accuracy to a matrix obtained from analytical expressions, can be prohibitively time consuming. For example, to construct $\underline{\underline{R}}_{T H}$ for a power MESFET with 10 fingers, each divided into active device elements 5 times along both length and width, would require 250 individual simulations. At $\sim 1 / 2$ hour per simulation for a finite difference calculation [8], the time demands are heavy, even making full use of symmetry.

\subsubsection{Double Fourier Series Finite Element Method}

To treat surface metallisation on a MMIC die, vertical matching of rectangular surface elements was described in Section 4.4. However, there is an approximation inherent in this approach, due to the imposition of adiabatic side wall boundary conditions on the surface elements. These conditions mean that no direct heat flow will occur between adjoining surface metal areas. Generally this is not expected to have much impact on calculated thermal resistance matrices, as the heat flow through the much greater cross sectional area of the MMIC die itself is unaffected by the 
adiabatic side wall boundary conditions on the metal elements. Also, any adjoining areas of metallisation can always be chosen to lie in low temperature regions away from centres of the active devices, so that the impact on heat flow of artificial adiabatic barriers will be small.

However, at the expense of increased algebraic complexity, the analytical thermal resistance matrix approach immediately generalises to remove this approximation. The thermal description of a cuboid with arbitrary fluxes defined on all 6 faces is given by the general solution

$$
\begin{aligned}
& \theta=c_{0}+c_{x} x+c_{y} y+c_{z} z+c_{x x} x^{2}+c_{y y} y^{2}+c_{z z} z^{2} \\
& +\sum_{m n}^{\prime}\left\{\begin{array}{c}
\cos \lambda_{m} x \cos \mu_{n} y \\
\times\left(A_{m n} \cosh \gamma_{m n} z+B_{m n} \sinh \gamma_{m n} z\right) \\
+\quad \cos \mu_{m} y \cos \gamma_{n} z \\
\times\left(C_{m n} \cosh \lambda_{m n} x+D_{m n} \sinh \lambda_{m n} x\right) \\
+\quad \cos \lambda_{m} x \cos \gamma_{n} z \\
\times\left(E_{m n} \cosh \mu_{m n} y+F_{m n} \sinh \mu_{m n} y\right)
\end{array}\right\},
\end{aligned}
$$

where

$$
\begin{gathered}
c_{x x}+c_{y y}+c_{z z}=0 \\
\lambda_{n}=\frac{n \pi}{L}, \mu_{n}=\frac{n \pi}{W}, \gamma_{n}=\frac{n \pi}{D}, \\
\lambda_{m n}^{2}=\mu_{m}^{2}+\gamma_{n}^{2}, \gamma_{m n}^{2}=\lambda_{m}^{2}+\mu_{n}^{2}, \mu_{m n}^{2}=\lambda_{m}^{2}+\gamma_{n}^{2} .
\end{gathered}
$$

Constructing this solution explicitly, interface matching can then be implemented not just vertically but also horizontally, avoiding the construction of artificial adiabatic barriers between adjoining sections of surface metal.

For simple cuboids with uniform surface fluxes $P_{1}, P_{2}, \ldots, P_{6}$ the solution reduces to just $x, y, z$ and $x^{2}, y^{2}, z^{2}$ terms, which gives a simple formulation of a finite element approach based on interface matching of primitive cuboids. This allows ready treatment of MMICs and of hybrid MICs with arbitrarily detailed surface metallisation.

\subsection{MMIC with Vias}

In Section 4.1, a simple analytical expression was derived for the thermal resistance matrix of a homogeneous MMIC. In this section it is shown how to derive the thermal resistance matrix for a MMIC containing an arbitrary distribution of full or partial thickness vias, or with partial substrate thinning.

Analytical solution for a single via has been presented previously [58], but this solution assumed a periodic distributions of vias, so that simple adiabatic boundary conditions could be imposed on a surface enclosing each via. For the MMIC and hybrid MIC case considered here, no such periodicity will generally exist.

Bonani et al., have presented approximations both for vias and for partial substrate thinning. Their description of vias is particularly simple and effective, and represents the MMIC with vias by means of the thermal resistance matrix for the corresponding homogeneous MMIC, with parallel resistances constructed for stand-alone metal vias, to take account of the greatly increased thermal conductivity of the via metal compared to the GaAs die [1-3].

Though vias have been shown to be largely unimportant in thermal simulations [4], (but not totally insignificant [1]), it may be the case that the thermal effects of vias could become particularly relevant with joint developments in via technology and development of accurate design tools. Partial substrate thinning, by contrast, is known to have a major impact on calculated MMIC temperatures $[1-3]$.

Increases in available computer memory and processor speed mean that it is now possible to construct and utilise analytical solutions which would previously have been of no practical value. The analytical solutions presented here require inversion and diagonalisation of large matrices (of order up to several $10^{3} \times 10^{3}$ ). Such solutions are therefore vastly more computationally expensive than the simple resistance matrix approximations of Bonani et al., but they represent an analytical 
solution that is numerically exact when fully converged, and that becomes increasingly more tractable with increased computing power. The computational time required to construct such accurate thermal resistance matrix descriptions has no impact on coupled electro-thermal run times, as the resistance matrices are calculated prior to coupled electro-thermal solution. The analytical solution for full thickness vias generalises to provide a solution for partial thickness vias or partial substrate thinning. These solutions, and construction of the corresponding thermal resistance matrices, are now described.

To the authors' best knowledge, this section presents the first analytical solution for the 3dimensional temperature distribution in a cuboid MMIC with an arbitrary distribution of vias of arbitrary cross-section (full or partial thickness).

\subsubsection{Formulation of the Linearised Problem}

By expressing the total thermal conductivity as the sum of GaAs MMIC conductivity, plus an additional contribution in the vicinity of each via, $j=1, \ldots, N$, the steady-state heat diffusion equation for a MMIC containing $N$ vias can be written

$$
\begin{gathered}
\nabla \cdot\left[\left\{\kappa(T)+\sum_{j} H_{j}(x, y)\left[\Delta \kappa_{j}(T)\right.\right.\right. \\
\left.\left.\left.+\delta \kappa_{j}(T)\right]\right\} \nabla T\right]=0
\end{gathered}
$$

where $T$ is physical temperature; $\kappa(T)$ is the temperature dependent thermal conductivity of the GaAs MMIC; $\Delta \kappa_{j}(T)$ for $j=1, \ldots, N$ are the (large) differences between the thermal conductivities of the metal vias and the $\mathrm{GaAs}$ MMIC, assuming the same functional form for temperature dependence as $\kappa(T) ; \quad \delta \kappa_{j}(T)$ for $j=1, \ldots, N$ are the small perturbations on $\Delta \kappa_{j}(T)$ due to the difference in temperature dependence of the via metal thermal conductivity from $\kappa(T)$; and

$$
H_{j}(x, y)= \begin{cases}1 & \text { in the region of via } j \\ 0 & \text { otherwise. }\end{cases}
$$

Then neglecting the small quantities $\delta \kappa_{j}(T)$, this equation can be written

$$
\nabla \cdot[\kappa(T) \nabla T]=-\nabla \cdot\left[\sum_{j} H_{j}(x, y) \Delta \kappa_{j}(T) \nabla T\right] .
$$

So the heat diffusion equation for the MMIC with vias has been converted into an equation with uniform GaAs MMIC thermal conductivity $\kappa(T)$, but with a temperature dependent volume heat sink term, described by the right-hand side of Eq. (55).

The justification for dropping the terms $\delta \kappa_{j}(T)$ is discussed in Subsection 4.5.5 below.

Equation (55) is non linear in $T$. To linearise the equation, the Kirchhoff transformation is performed [55] giving

$$
\nabla^{2} \theta=-\nabla \cdot\left[\sum_{j} \xi_{j} H_{j}(x, y) \nabla \theta\right]
$$

with the $\xi_{j}$ defined in Subsection 4.5 .5 below.

In terms of linearised temperature, $\theta$, the boundary conditions become

$$
\begin{gathered}
\left.\frac{\partial \theta}{\partial x}\right|_{x=0, L}=0 \\
\left.\frac{\partial \theta}{\partial y}\right|_{y=0, W}=0 \\
\theta(z=D)=T_{S} \\
\left.\kappa_{S} \frac{\partial \theta}{\partial z}\right|_{z=0}=-\sum_{i} \frac{S_{i}(x, y)}{1+\sum_{j} \xi_{j} H_{j}(x, y)} P_{i}
\end{gathered}
$$

where

$$
S_{i}(x, y)= \begin{cases}1 & \text { for }(x, y) \in D_{i} \\ 0 & \text { otherwise }\end{cases}
$$




\subsubsection{Double Cosine Transform}

To solve this problem the double Fourier series solution of Eq. (14) is generalised to

$$
\theta=\sum_{m n} \cos \lambda_{m} x \cos \mu_{n} y Z_{m n}(z),
$$

where

$$
\begin{aligned}
\lambda_{m} & =\frac{m \pi}{L}, \\
\mu_{n} & =\frac{n \pi}{W}
\end{aligned}
$$

and $m, n=0,1,2, \ldots$.

It is apparent that Eq. (62) immediately satisfies the adiabatic $x$ - and $y$-flux boundary conditions given by Eqs. (57) and (58). Substituting the $z$ direction boundary conditions, Eqs. (59) and (60), into solution Eq. (62) provides conditions on the $Z_{m n}$,

$$
\left.Z_{m n}\right|_{z=D}=T_{S} \delta_{0 m} \delta_{0 n}
$$

and

$$
\left.\frac{d Z_{m n}}{d z}\right|_{z=0}=\frac{-4}{\kappa_{S} L W\left(1+\delta_{m 0}\right)\left(1+\delta_{n 0}\right)} \sum_{i} I_{m n}^{i} P_{i}
$$

Equations (66) and (23) assume that no elementary heating element, $D_{i}$, ever lies totally or partially over a via. For semiconductor active device channels and full thickness metal vias this will always be true, but the extension to more general surface heating elements, partial thickness vias and partial substrate thinning is immediate.

Finally, performing a double cosine transformation of Eq. (56) [9] gives

$$
0=\sum_{(m n)} A_{\left(m^{\prime} n^{\prime}\right)(m n)} \frac{d^{2} Z_{(m n)}}{d z^{2}}-B_{\left(m^{\prime} n^{\prime}\right)(m n)} Z_{(m n)},
$$

where

$$
\begin{aligned}
A_{\left(m^{\prime} n^{\prime}\right)(m n)}= & \frac{L W}{4}\left(1+\delta_{m^{\prime} 0}\right)\left(1+\delta_{n^{\prime} 0}\right) \delta_{m m^{\prime}} \delta_{n n^{\prime}} \\
& +\sum_{j} \xi_{j} I_{m m^{\prime}}^{j x} I_{n n^{\prime}}^{j y}
\end{aligned}
$$

and

$$
\begin{aligned}
& B_{\left(m^{\prime} n^{\prime}\right)(m n)} \\
& =\frac{L W}{4}\left(1+\delta_{m^{\prime} 0}\right)\left(1+\delta_{n^{\prime} 0}\right) \delta_{m m^{\prime}} \delta_{n n^{\prime}}\left(\lambda_{m^{\prime}}^{2}+\mu_{n^{\prime}}^{2}\right)+\sum_{j} \xi_{j} \\
& \times\left[\begin{array}{l}
\left(\lambda_{m}^{2}+\mu_{n}^{2}\right) I_{m m^{\prime}}^{j x} I_{n n^{\prime}}^{j y} \\
+I_{n n^{\prime}}^{j y} \lambda_{m} \times \\
\left(\cos \lambda_{m^{\prime}} x_{j}^{L} \sin \lambda_{m} x_{j}^{L}-\cos \lambda_{m^{\prime}} x_{j}^{R} \sin \lambda_{m} x_{j}^{R}\right) \\
+I_{m m^{\prime}}^{j x} \mu_{n} \times \\
\left(\cos \mu_{n^{\prime}} y_{j}^{D} \sin \mu_{n} y_{j}^{D}-\cos \mu_{n^{\prime}} y_{j}^{U} \sin \mu_{n} y_{j}^{U}\right)
\end{array}\right]
\end{aligned}
$$

and where, for simplicity, the vias have been assumed to have uniform rectangular cross-section with faces parallel to the faces of the MMIC. $x_{j}^{L}, x_{j}^{R}$ are the $x$-coordinates of the edges of via $j$ parallel to the $y$-axis, and $y_{j}^{D}, y_{j}^{U}$ are the $y$-coordinates of the edges of via $j$ parallel to the $x$-axis. The generalisation to vias of arbitrary uniform cross-section is immediate.

Elementary integrals $I_{m m^{\prime}}^{j x}, I_{n n^{\prime}}^{j y}$ have been defined as

$$
\begin{aligned}
I_{m m^{\prime}}^{j x} & \equiv \int_{x_{j}^{L}}^{x_{j}^{R}} \cos \lambda_{m} x \cos \lambda_{m^{\prime}} x d x \\
I_{n n^{\prime}}^{j y} & \equiv \int_{y_{j}^{D}}^{y_{j}^{U}} \cos \mu_{n} y \cos \mu_{n^{\prime}} y d y
\end{aligned}
$$

and are simple to evaluate analytically.

The linearised partial differential equation for the GaAs MMIC with vias, Eq. (56), with boundary conditions Eqs. (57)-(60), has thus been converted to a system of coupled, linear, 2nd order, ordinary differential equations, Eq. (67), for the $Z_{m n}$ of Eq. (62), with boundary conditions given by Eqs. (65) and (66).

For the special case of $\xi_{j}=0$ for all $j$, corresponding to a MMIC with no vias, this system of equations just reduces to

$$
\frac{d^{2} Z_{m n}}{d z^{2}}=\gamma_{m n}^{2} Z_{m n}
$$


where $\gamma_{m n}^{2}=\lambda_{m}^{2}+\mu_{n}^{2}$, with general solution

$Z_{m n}=\left\{\begin{array}{l}A+B z, \quad \text { for }(m, n)=(0,0) \\ C_{m n} \cosh \gamma_{m n} z+S_{m n} \sinh \gamma_{m n} z, \quad \text { otherwise }\end{array}\right.$

corresponding to the usual separation of variables solution.

\subsubsection{Solution of the Coupled Linear System}

In matrix notation, the coupled system of equations, Eq. (67) can be expressed

$$
\frac{d^{2} \underline{Z}}{d z^{2}}-\underline{\underline{A}}{ }^{-1} \underline{\underline{B}} \underline{Z}=\underline{0} .
$$

Examination of Eqs. (68) and (69) shows that, in general, all elements of matrix $\underline{\underline{A}}$ will be non zero. For the case of a MMIC with no vias, $\underline{\underline{A}}$ will be diagonal with all diagonal elements non zero. However, although the matrix elements of matrix $\underline{\underline{B}}$ are mostly non zero in the general case, putting $(m, n)=(0,0)$ in Eq. $(69)$ means that $B_{\left(m^{\prime} n^{\prime}\right)(00)}$ is seen to be zero for all $\left(m^{\prime}, n^{\prime}\right)$. For the case of a MMIC with no vias, $\underline{\underline{B}}$ is diagonal with one diagonal element equal to zero.

Writing $\underline{A}^{-1}, \underline{B}$ and $\underline{Z}$ in partitioned form, with (double) index $\left(m^{\prime}, n^{\prime}\right)=(0,0)$ corresponding to the first row and $(m, n)=(0,0)$ corresponding to the first column,

$$
\begin{aligned}
& \underline{\underline{A}}^{-1}=\left(\begin{array}{ll}
A_{11}^{-1} & \underline{A^{-1}} 12 \\
\underline{A^{-1}} 21 & \underline{\underline{A^{-1}}} 22
\end{array}\right), \\
& \underline{\underline{B}}=\left(\begin{array}{ll}
0 & \underline{B}_{12}^{T} \\
\underline{0} & \underline{\underline{B}}_{22}
\end{array}\right), \\
& \underline{Z}=\left(\begin{array}{l}
Z_{00} \\
\underline{Z}^{\prime}
\end{array}\right) .
\end{aligned}
$$

Then partitioning $\underline{\underline{A}}^{-1} \underline{\underline{B}}$ in the same way as above

$$
\left(\begin{array}{c}
d^{2} Z_{00} / d z^{2} \\
d^{2} \underline{Z}^{\prime} / d z^{2}
\end{array}\right)=\left(\begin{array}{ll}
0 & \underline{A^{-1} B} \\
12 \\
\underline{0} & \underline{\underline{A^{-1} B_{1}}}
\end{array}\right)\left(\begin{array}{c}
Z_{00} \\
\underline{Z}^{\prime}
\end{array}\right) .
$$

This gives rise to the closed set of equations for $\underline{Z}^{\prime}$, i.e., for all the $Z_{m n}$ except $Z_{00}$,

$$
\frac{d^{2} \underline{Z}^{\prime}}{d z^{2}}-\underline{\underline{A^{-1} B}} 22 \underline{Z}^{\prime}=\underline{0},
$$

subject to the boundary conditions

$$
\left.\underline{Z}^{\prime}\right|_{z=D}=\underline{0}
$$

and Eq. (66) for all $m, n$ excluding $(m, n)=(0,0)$.

Solving the homogeneous linear system for $\underline{Z}^{\prime}$ then gives the inhomogeneous equation for $Z_{00}$,

$$
\frac{d^{2} Z_{00}}{d z^{2}}=\underline{A}^{-1} B_{12}^{T} \underline{Z}^{\prime}
$$

subject to the boundary conditions

$$
\begin{gathered}
\left.Z_{00}\right|_{z=D}=T_{S} \\
\left.\frac{d Z_{00}}{d z}\right|_{z=0}=\frac{-1}{\kappa_{S} L W} \sum_{i} I_{00}^{i} P_{i} .
\end{gathered}
$$

The coupled system for $\underline{Z}^{\prime}$, Eq. (79), can be solved by following the standard procedure for systems of second order linear differential equations with constant coefficients [59]. Define

$$
\underline{G} \equiv\left(\begin{array}{c}
\underline{Z}^{\prime} \\
d \underline{Z}^{\prime} / d z
\end{array}\right)
$$

then it is immediately obvious that,

$$
\frac{d \underline{G}}{d z}=\underline{\underline{M}} \underline{G}
$$

where

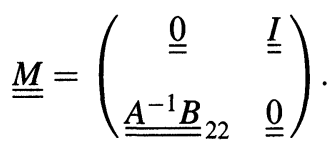




\section{Writing}

$$
\underline{G}=e^{\gamma z} \underline{u}
$$

where from the definition of Eq. (84)

$$
\underline{u}=\left(\begin{array}{c}
\underline{u}^{I} \\
\gamma \underline{u}^{I}
\end{array}\right)
$$

and $\underline{u}^{I}$ is independent of $z$, Eq. (85) gives the eigenvalue problem

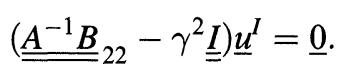

For the zero via case, the $\gamma^{2}$ are pure real and positive definite, going as $\lambda_{m}^{2}+\mu_{n}^{2}$ with $(m, n) \neq(0,0)$. However, the eigenproblem of Eq. (89) is constructed for a non symmetric real

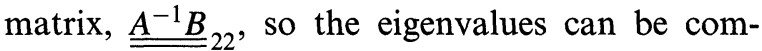
plex. Although not obvious for the most general case, consideration of the terms of the matrices $\underline{\underline{A}}$ and $\underline{B}$ of Eqs. (68) and (69) shows that typically the additional terms due to vias will be a small perturbation on the positive definite diagonal terms of $\underline{\underline{A}}_{22}$ corresponding to $\lambda_{m}^{2}+\mu_{n}^{2}$. It is therefore to be expected that the modified eigenvalues of ${\underline{\underline{A^{-1} B}}}_{22}$ will remain real and positive in the presence of vias. This is found to be the case for all calculations performed so far. In the general case $\operatorname{det}\left({\underline{A^{-1} B}}_{22}\right)$ will be non zero, so that $\gamma$ is never zero (for non trivial solutions $\underline{u}^{I}$ ).

Solving Eq. (89) by standard numerical techniques, to obtain eigenvalues $\gamma_{j}^{2}$ and eigenvectors $\underline{u}_{j}^{I}$, the general solution to Eq. (85) can be written

$$
\underline{Z}^{\prime}=\sum_{j}\left(c_{j}^{+} e^{+\gamma_{j} z}+c_{j}^{-} e^{-\gamma_{j} z}\right) \underline{u}_{j}^{I} .
$$

To obtain the unknown coefficients $c_{j}^{ \pm}$, the boundary conditions given by Eqs. (66) and (80) are imposed. The vectors $\underline{c}^{ \pm}$of components $c_{j}^{ \pm}$are then seen to satisfy

$$
\begin{gathered}
\underline{\underline{U_{\gamma}}}\left(\underline{c}^{+}-\underline{c}^{-}\right)=\underline{p}, \\
\underline{\underline{U_{+}}} \underline{\underline{c}}^{+}+\underline{\underline{U_{-}}} \underline{c}^{-}=\underline{0},
\end{gathered}
$$

where the columns, $\underline{U_{\gamma}} \dot{j}$, of matrix $\underline{\underline{U_{\gamma}}}$ are given by

$$
\underline{U_{\gamma}}=\gamma_{j} \underline{u}_{j}^{I}
$$

similarly

$$
\begin{aligned}
& {\underline{U_{+}}}_{j}=e^{+\gamma_{j} D} \underline{u}_{j}^{I}, \\
& \underline{U}_{-j}=e^{-\gamma_{j} D} \underline{u}_{j}^{I}
\end{aligned}
$$

and $\underline{p}$ is the vector with components

$$
\begin{array}{r}
p_{(m n)}=\frac{-4}{\kappa_{S} L W\left(1+\delta_{m 0}\right)\left(1+\delta_{n 0}\right)} \sum_{i} I_{m n}^{i} P_{i} \\
\text { for }(m, n) \neq(0,0) .
\end{array}
$$

Having constructed the solution for $\underline{Z}^{\prime}$ the solution for $Z_{00}$ is immediately obtained as

$$
Z_{00}=A+B z+\sum_{j} \frac{1}{\gamma_{j}^{2}}\left(\tilde{c}_{j}^{+} e^{+\gamma_{j} z}+\tilde{c}_{j}^{-} e^{-\gamma_{j} z}\right),
$$

where

$$
\tilde{c}_{j}^{ \pm}=c_{j}^{ \pm} \underline{A}^{-1} \underline{B}_{12}^{T} \underline{u}_{j}^{I}
$$

and arbitrary constants $A, B$ are determined by imposing boundary conditions, Eqs. (82) and (83).

This gives a complete analytical solution for the case of a cuboid MMIC with an arbitrary distribution of vias. The major computational limitation on this approach is the order of the matrix problems required for full convergence of the double Fourier series expansion, Eq. (62). For a MMIC with no vias, adequate convergence is typically found to be obtained for $m, n=0, \ldots, \sim 50$, dependent on the size of the smallest feature to be resolved relative to the die size $[10,11]$. The inclusion of vias is often found to be a small perturbation on the MMIC solution $[1,4]$, so similar convergence rates might be expected for the solution with vias. However, the effects of discontinuities in thermal conductivities is to introduce discontinuities into thermal 
gradients at the via interfaces. These gradient discontinuities could require larger number of terms for an accurately converged solution.

Assuming adequate convergence of Eq. (62) with $m^{2}+n^{2} \leq 50^{2}$ for the system under consideration, the size of the matrices to be constructed is of order $\sim 2000 \times 2000$, requiring $\sim 32 \mathrm{MB}$ of real double precision storage for each matrix. Inversion, diagonalisation and multiplication of matrices of this size typically takes $\sim 1$ hour to $\sim 10$ hours.

\subsubsection{Thermal Resistance Matrix}

To construct the thermal resistance matrix, analytical expressions are required for the average temperatures over elementary heating elements $D_{i}$. From Eqs. (62) and (23) these are given by

$$
\begin{aligned}
\theta_{a v}^{i} & =\frac{\left.\iint_{D i} \theta\right|_{z=0} d x d y}{\iint_{D i} d x d y}, \\
& =\left.\sum_{m n} \frac{I_{m n}^{i}}{I_{00}^{i}} Z_{m n}\right|_{z=0},
\end{aligned}
$$

or in matrix notation,

$$
\theta_{a v}^{i}=\left.Z_{00}\right|_{0}+\left.\underline{\tilde{I}}^{i T} \underline{Z}^{\prime}\right|_{0}
$$

where

$$
\tilde{I}_{(m n)}^{i}=\frac{I_{m n}^{i}}{I_{00}^{i}}, \quad(m, n) \neq(0,0)
$$

From Eqs. (90)-(92),

$$
\left.\underline{Z}^{\prime}\right|_{0}=\underline{\underline{\Pi}} \underline{p}
$$

where

$$
\underline{\underline{\Pi}}=\underline{\underline{U}}\left(\underline{\underline{I}}-{\underline{\underline{U_{-}}}}^{-1} \underline{\underline{U_{+}}}\right)\left(\underline{\underline{I}}+{\underline{\underline{U_{-}}}}^{-1}{\underline{\underline{U_{+}}}}^{-1} \underline{\underline{U_{\gamma}}}\right.
$$

and where $\underline{\underline{U}}$ is the matrix with columns $\underline{U}_{j}=\underline{u}_{j}^{I}$. Hence,

$$
\theta_{a v}^{i}=\left.Z_{00}\right|_{0}+\underline{\tilde{I}}^{i T} \underline{\underline{\Pi}} \underline{p}
$$

and

$$
\theta_{a v}^{i}-\left.Z_{00}\right|_{0}=\sum_{k} R_{T H_{i k}} P_{k},
$$

where the thermal resistance matrix $\underline{\underline{R}}_{T H}$ is given by

$$
R_{T H_{i k}}=\underline{\tilde{I}}^{i T} \underline{\underline{\Pi}} \underline{\tilde{I}}^{k}
$$

with

$$
\tilde{\tilde{I}}_{(m n)}^{k}=\frac{-4}{\kappa_{S} L W\left(1+\delta_{m 0}\right)\left(1+\delta_{n 0}\right)} I_{m n}^{k} .
$$

As for the homogeneous case, the thermal resistance matrix is seen to be obtained purely in terms of material parameters, and the geometry of the MMIC. As it is derived for the linearised temperature, it is independent of both temperature and power density, and hence of electrical bias point (though parameters $\xi_{j}$ must be chosen to represent typical operating conditions). The matrix only has to be constructed once, for repeated use in coupled electro-thermal simulations, and whatever the complexity of the MMIC internal structure the order of the thermal resistance matrix is determined purely by the (small) number of surface heating elements, $D_{i}$.

\subsubsection{Non Linear Thermal Conductivity Perturbation}

In Subsection 4.5.1, terms $\delta \kappa_{j}(T)$ were dropped from the heat diffusion equation on the grounds that they were a small perturbation on terms $\Delta \kappa_{j}(T)$. This assertion is now justified. Also, in the linearised Eq. (56), the constants $\xi_{j}$ were introduced; these are now defined.

If the thermal conductivity in the GaAs MMIC body, region $I$, is $\kappa_{I}(T)$, and the thermal conductivity in the metal vias, region $I I$, is $\kappa_{I I}(T)$, (and for simplicity all vias are assumed to be made of identical material, though this is not a necessary restriction), total thermal conductivity throughout the whole MMIC, $\kappa_{\text {tot }}(T)$, 
can be written

$$
\kappa_{\mathrm{tot}}(T)=\kappa_{I}(T)+\sum_{j} H_{j}(x, y)\left[\kappa_{I I}(T)-\kappa_{I}(T)\right] .
$$

To a good approximation, the thermal conductivity of GaAs can be assumed to have a simple power law dependence on physical temperature over temperature ranges of interest for device operation [56], and the thermal conductivity of via metal can be assumed independent of temperature [7],

$$
\begin{gathered}
\kappa_{I}(T)=\kappa_{I}\left(\frac{T}{T_{S}}\right)^{-b} \quad b=1.22 \text { for GaAs, } \\
\kappa_{I I}(T)=\kappa_{I I} \quad \text { for via metal. }
\end{gathered}
$$

Then the $\Delta \kappa_{j}(T)$ and $\delta \kappa_{j}(T)$ for each via, $j=1, \ldots, N$, can be defined by the relations,

$$
\kappa_{I I}(T)-\kappa_{I}(T)=\Delta \kappa_{j}(T)+\delta \kappa_{j}(T),
$$

where

$$
\Delta \kappa_{j}(T) \equiv\left(\eta_{j} \kappa_{I I}-\kappa_{I}\right)\left(\frac{T}{T_{S}}\right)^{-b} \equiv \xi_{j} \kappa_{I}(T)
$$

which defines the $\xi_{j}$, and

$$
\delta \kappa_{j}(T)=\kappa_{I I}-\left(1+\xi_{j}\right) \kappa_{I}(T) .
$$

$\Delta \kappa_{j}(T)$ of Eq. (113) equals the difference, $\kappa_{I I}(T)-\kappa_{I}(T)$, assuming the same functional form for the temperature dependences of $\kappa_{I I}(T)$ and $\kappa_{I}(T)$. Equation (114) then gives the small perturbation on $\Delta \kappa_{j}(T)$ resulting from the fact that the temperature dependences of $\kappa_{I I}(T)$ and $\kappa_{I}(T)$ are actually of different functional form. This separation of $\Delta \kappa_{j}(T)$ and $\delta \kappa_{j}(T)$ contributions to $\kappa_{I I}(T)-\kappa_{I}(T)$ is necessary to allow linearisation of Eq. (55) by use of the Kirchhoff transformation, Eq. (9).

The constants $\eta_{j}$ (and hence $\xi_{j}$ ) in Eq. (113) are introduced to allow minimisation of the errors introduced by dropping terms $\delta \kappa_{j}(T)$ from
Eq. (53). Although $\kappa_{I I}(T)-\kappa_{I}(T)$ is the same in each via, these constants are given different values in each via, $\eta_{j}$ (and so $\xi_{j}$ ) $j=1, \ldots, N$, to allow for the fact that the temperature distribution will differ from via to via. The use of the $\xi_{j}$, in minimisation of the error due to neglect of differences between the functional forms of the temperature dependences of the thermal conductivities, is now discussed. The same general approach applies to hierarchical construction of thermal resistance matrices for metal on MMIC structures, as described in Section 4.4.

The magnitude of the perturbation $\delta \kappa_{j}(T)$ on $\Delta \kappa_{j}(T)$ is given by

$$
\frac{\delta \kappa_{j}(T)}{\Delta \kappa_{j}(T)}=\frac{1}{\xi_{j}}\left[\frac{\kappa_{I I}}{\kappa_{I}}\left(\frac{T}{T_{S}}\right)^{b}-\left(1+\xi_{j}\right)\right] .
$$

This is seen to be exactly zero for the case that the square bracket on the right hand side of Eq. (115) goes to zero. So defining the $\xi_{j}$ by

$$
1+\xi_{j}=\frac{\kappa_{I I}}{\kappa_{I}}\left(\frac{T_{\mathrm{via}_{j}}}{T_{S}}\right)^{b},
$$

for typical via temperatures $T_{\mathrm{via}_{j}}$, and writing the temperature variation over via $j$ as $T=T_{\text {via }_{j}}$ $\pm \Delta T_{j}$

$$
\begin{aligned}
\frac{\delta \kappa_{j}(T)}{\Delta \kappa_{j}(T)}= & \frac{1}{\left[1-\left(\kappa_{I} / \kappa_{I I}\right)\left(T_{S} / T_{\mathrm{via}_{j}}\right)^{b}\right]} \\
& \times\left[\left(1 \pm \frac{\Delta T_{j}}{T_{\mathrm{via}_{j}}}\right)^{b}-1\right]
\end{aligned}
$$

which is given approximately by

$$
\frac{\delta \kappa_{j}(T)}{\Delta \kappa_{j}(T)} \approx \pm 1.4 \frac{\Delta T_{j}}{T_{\mathrm{via}_{j}}},
$$

for a typical ratio $\kappa_{I} / \kappa_{I I}$ of $\sim 1 / 8$.

For a variation across a via of $\pm 10 \mathrm{~K}$, from a typical via temperature of $\sim 300 \mathrm{~K}$, this error is $\delta \kappa_{j}(T) / \Delta \kappa_{j}(T) \approx \pm 5 \%$. The impact on calculated active device temperatures, due to neglect of errors of this magnitude in the via thermal conductivities, is expected to be small. 
Clearly, mean via temperatures $T_{\text {via }}$ are not known a priori, so they have to be obtained selfconsistently, for instance by relaxation starting with temperature values obtained from the simple analytical solution for the MMIC without vias. This self-consistent solution for the $\xi_{j}$ then gives an approximate treatment of the non linear perturbation arising from the difference in functional form between the temperature dependences of the thermal conductivities in the GaAs MMIC and in the metal vias.

As the metal vias are of small cross-sectional area, and have high thermal conductivities, the greatest variation in temperature is expected to be along the $z$-axis, corresponding to the heat flux from the active devices at the MMIC surface to the heat sink mount at the MMIC base. The accuracy of the solution can therefore be improved further, by subdividing the vias in the $z$-direction. By generalising the mean via temperature description, $T_{\mathrm{via}_{j}}$, and solving self-consistently for mean via temperatures, $T_{\mathrm{via}_{j k}}$, in each vertical element, $k$, of any given via, $j$, typical temperature variations across via elements, $\Delta T_{j k}$, can be reduced. The description of the vias, $j=1, \ldots, N$, already allows for the possibility that the cross-section of each physical via can be constructed from mathematical sub-vias of smaller cross-section. Combined with the generalisation to vertical subelements, $k$, described by mean temperatures $T_{\text {via }_{j k}}$, this means that the variation of temperatures across elementary via volumes, $\Delta T_{j k}$, can be made arbitrarily small giving an essentially numerically exact selfconsistent solution to the full non linear problem of a MMIC with vias. However, this full solution is very computationally demanding and generally not warranted by the degree of inaccuracy due to the non linear perturbation.

In a fully coupled electro-thermal solution, it would be impractical to obtain the fully non linear solution for the MMIC thermal resistance matrix at each iteration of a global electro-thermal solution. However, by obtaining the thermal resistance matrix for a typical operating point, the resulting linear description of the MMIC should be sufficiently accurate for description of the global system over a satisfactory range of operation.

Figure 9 shows the calculated temperature distribution of a GaAs MMIC bearing two, 6finger, power transistors. Figure 10 shows the same die with a central via. For speed of calculation, these illustrative simulations used just a small number of basis states given by the relation $m^{2}+n^{2} \leq 20^{2}$. The details of the individual device fingers are therefore not resolved, but the impact of the centrally placed via is clear, reducing central temperature and modifying the temperatures in the vicinity of the active device fingers.

\subsubsection{Partial Thickness Vias and Substrate Thinning}

To treat the case of partial thickness vias and partial substrate thinning, and to allow more accurate minimisation of non linear perturbations, $\delta \kappa_{j}(T)$, the extension to vias discretised along the $z$-axis is now presented. The linearised heat diffusion equation, Eq. (56), becomes

$$
\nabla^{2} \theta=-\nabla \cdot\left[\sum_{j k} \xi_{j k} H_{j}(x, y) H_{k}(z) \nabla \theta\right]
$$

where

$$
H_{k}(z)= \begin{cases}1, & \text { within MMIC layer } k \\ 0, & \text { otherwise }\end{cases}
$$

Then the coupled system for the $Z_{m n}$ of Eq. (67) becomes

$$
\frac{d}{d z}\left[\underline{\underline{A}}(z) \frac{d \underline{Z}}{d z}\right]-\underline{\underline{B}}(z) \underline{Z}=\underline{0},
$$

where $\underline{\underline{A}}$ and $\underline{\underline{B}}$ are now piecewise constant and have the same form as previously within each MMIC layer, Eqs. (68) and (69), but with $\sum_{j} \xi_{j} \rightarrow \sum_{j k} \xi_{j k} H_{k}(z)$. Integrating Eq. (121) across an interface, it is seen to reduce to the original Eq. (67) within each layer, with continuity of 


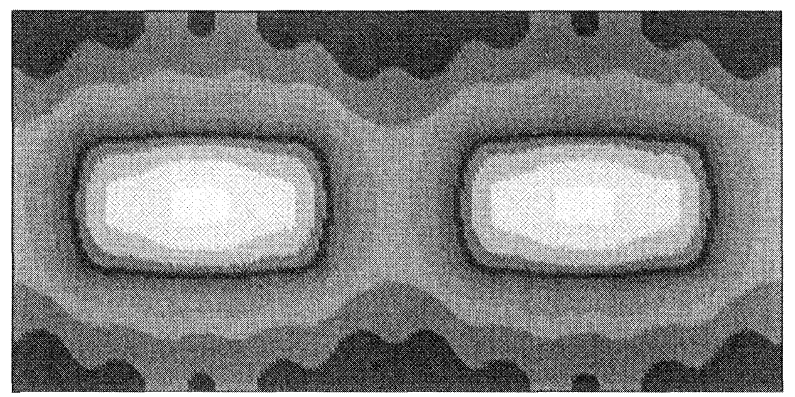

FIGURE 9 Simulation of a $500 \mu \mathrm{m} \times 250 \mu \mathrm{m}$ GaAs MMIC bearing two 6-finger power transistors dissipating $0.3 \mathrm{~W}$ total.

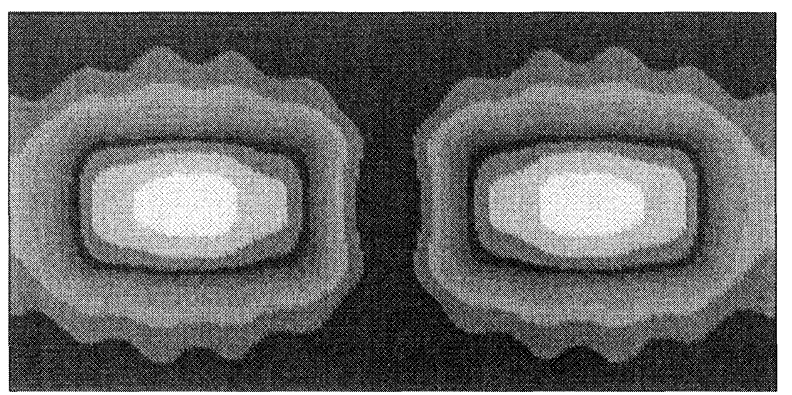

FIGURE 10 Simulation of same MMIC as Figure 9 but with a $50 \mu \mathrm{m} \times 50 \mu \mathrm{m}$ central via $15^{\circ} \mathrm{C}$ above heat sink mount (peak) to $5^{\circ} \mathrm{C}$ above mount (edge).

$\underline{Z}$ and $\underline{A} d \underline{Z} / d z$ across each interface. This problem can then be treated by a transfer matrix approach.

The above formulation can also, in principle, treat the case of totally arbitrary surface metallisation considered as an effective partial thickness via of complicated cross-section. However, treatment of surface detail that is too fine will generally produce convergence difficulties. Also, this approach is more limited in its description of non linear interface matching between metal and GaAs.

\subsubsection{Alternative Formulation}

After some manipulation, Eq. (56) can be written in the equivalent form,

$$
\nabla^{2} \theta=\sum_{j} \ln \left(1+\xi_{j}\right) \nabla H_{j}(x, y) \cdot \nabla \theta
$$

as long as $\xi_{j}>-1$ for all $j$. This form again gives rise to Eq. (67) with matrix $\underline{B}$ of similar form to Eq. (69), but now matrix $\underline{\underline{A}}$ is diagonal and is trivial to invert. This represents a significant saving in the construction of $\underline{\underline{A}}^{-1} \underline{\underline{B}}$ when the order of matrix $\underline{\underline{A}}$ is large. $\underline{\underline{A}}^{-1} \underline{\underline{B}}$ is given by

$$
\begin{aligned}
A^{-1} B_{\left(m^{\prime} n^{\prime}\right)(m n)} & =\left(\lambda_{m^{\prime}}^{2}+\mu_{n^{\prime}}^{2}\right) \delta_{m m^{\prime}} \delta_{n n^{\prime}} \\
& +\sum_{j} \ln \left(1+\xi_{j}\right) \frac{4}{L W\left(1+\delta_{m^{\prime} 0}\right)\left(1+\delta_{n^{\prime} 0}\right)} \\
& \times\left[\begin{array}{l}
+I_{n n^{\prime}}^{j y} \lambda_{m} \times \\
\left(\cos \lambda_{m^{\prime}} x_{j}^{L} \sin \lambda_{m} x_{j}^{L}-\cos \lambda_{m^{\prime}} x_{j}^{R} \sin \lambda_{m} x_{j}^{R}\right) \\
+I_{m m^{\prime}}^{j x} \mu_{n} \times \\
\left(\cos \mu_{n^{\prime}} y_{j}^{D} \sin \mu_{n} y_{j}^{D}-\cos \mu_{n^{\prime}} y_{j}^{U} \sin \mu_{n} y_{j}^{U}\right)
\end{array}\right] .
\end{aligned}
$$




\subsubsection{Approximate Treatment of Vias}

The double Fourier series solutions for the treatment of vias, presented above, are computationally demanding. However, the approximate equivalence principle approach of Bonani et al. $[1-3]$ is conceptually simple and is cheap to implement. It is described here within the framework of the resistance matrix formulation.

Partitioning the thermal resistance matrix equation, Eq. (7), by elementary surface elements corresponding to active devices, $\underline{\theta}_{a}, \underline{P}_{a}$ and via holes, $\underline{\theta}_{v}, \underline{P}_{v}$, gives

$$
\left(\begin{array}{l}
\underline{\Delta \theta}_{a} \\
\underline{\Delta \theta}_{v}
\end{array}\right)=\left(\begin{array}{ll}
\underline{\underline{R}}_{T H}^{a a} & \underline{\underline{R}}_{T H}^{a v} \\
\underline{\underline{R}}_{T H}^{v a} & \underline{\underline{R}}_{T H}^{v v}
\end{array}\right)\left(\begin{array}{l}
\underline{P}_{a} \\
\underline{P}_{v}
\end{array}\right),
$$

with

$$
\underline{\theta \theta}_{v}=R_{T H}^{\mathrm{via}}\left(-\underline{P}_{v}\right)
$$

describing the fluxes through the parallel equivalent via thermal resistances, $R_{T H}^{\text {via }}$.

All vias are assumed identical for simplicity. The negative sign in Eq. (125) reflects the fact that the parallel resistances, corresponding to the greater thermal conductivity of the metal compared to $\mathrm{GaAs}$ and applying the equivalence principle, are all effectively external to the GaAs die. (This means that although the parallel resistances describe increased heat flow through via metal in an approximate fashion, they do not correctly include the effects of increased heat flow through the side walls of the via metal in the GaAs.)

Combining Eqs. (124) and (125), the global thermal resistance matrix relating active device temperature rises to active device power dissipations is given by,

$$
\underline{\Delta \theta}_{a}=\underline{\underline{R}}_{T H}^{\mathrm{glob}} \underline{P}_{a}
$$

with

$$
\underline{\underline{R}}_{T H}^{\text {glob }}=\underline{\underline{R}}_{T H}^{a a}-\underline{\underline{R}}_{T H}^{a v}\left(\underline{\underline{R}}_{T H}^{v v}+\underline{\underline{I}}_{T H}^{\mathrm{via}}\right)^{-1} \underline{\underline{R}}_{T H}^{v a}
$$

For a via of uniform cross-section, passing through a GaAs die of thickness, $D$, with difference between metal and GaAs thermal conductivity given by, $\Delta \kappa$, the via thermal resistance is given simply by

$$
R_{T H}^{\mathrm{via}}=\frac{D}{\Delta \kappa}
$$

To describe more accurately the thermal non linearity due to the difference in functional form between the temperature dependent thermal conductivities of metal and GaAs, $\Delta \kappa$ can be ascribed a different value in each via, corresponding to respective mean temperature.

Combining this approach with a simple generalisation of the 2-layer thermal resistance matrix solution of Section 4.3 provides a straightforward description of partial thickness vias and partial substrate thinning. In the case of partial substrate thinning or 'bathtubs' [1], flux at the surface of the partial thickness bathtub metallisation is assumed piecewise constant, rather than completely uniform.

\subsection{Large Area Substrates}

For large area substrates, from MMIC grid arrays up to circuit board level, surface fluxes due to convection and radiation must be included in the solution of the heat diffusion equation. (The same would hold for small area substrates under any circumstances in which convection became important.) For moderately low power densities and temperatures these fluxes can be described by the linear radiation boundary condition, Eq. (13) [19]. Putting $\alpha_{0, D}=1, H_{0, D} \neq 0$ the steady-state heat diffusion equation, Eq. (10), can be solved to obtain the corresponding thermal resistance matrix for a substrate with radiative and convective surface losses. Figures 11-13 show the results of electro-thermal simulations performed using such a thermal resistance matrix.

Putting $\alpha_{0}=\alpha_{D}=1,-H_{0}=H_{D}=H, \theta_{0}(x, y)=$ $\theta_{D}(x, y)=\theta_{0}, p_{D}(x, y)=0$ and $p_{0}(x, y)=\sum_{i} S_{i}(x, y)$ 


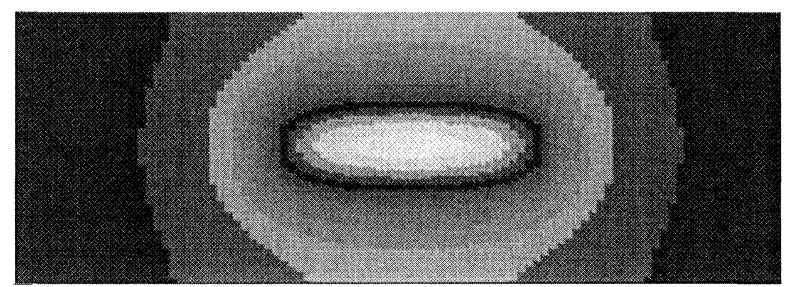

FIGURE 11 Electro-thermal simulation of Cu/FR-4 mount reverse bearing a balanced amplifier MMIC. Cooling entirely by radiation and convection (no heatsink). $46^{\circ} \mathrm{C}$ above ambient (centre) to ambient (edge) $[18,19]$.

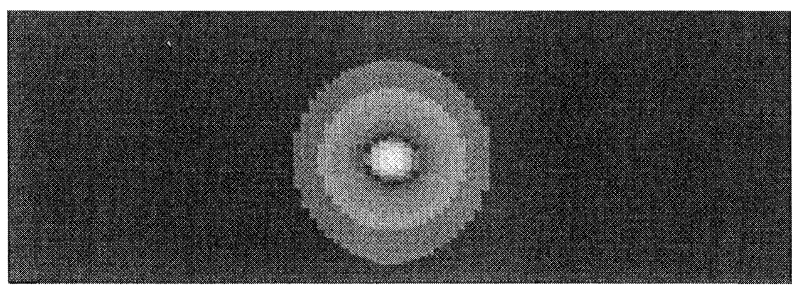

FIGURE 12 Simulated temperature of the FR-4 mount reverse side of Figure 11 neglecting Cu layer. $96^{\circ} \mathrm{C}$ above ambient (centre) to ambient (edge) [19].

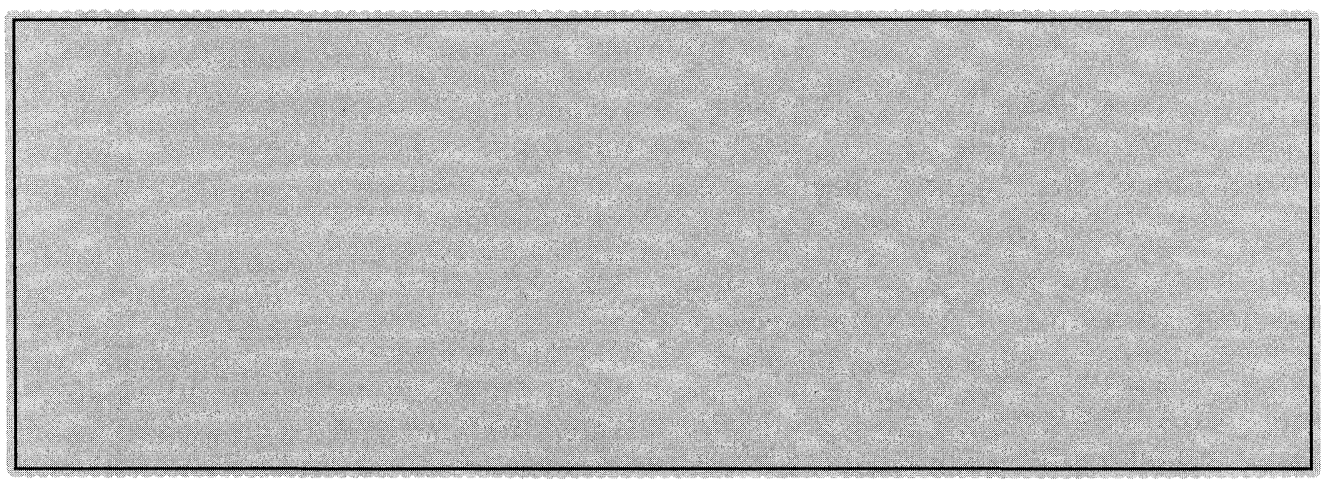

FIGURE 13 Simulated temperature of the FR-4 mount reverse side of Figure 11 assuming AlN parameters. $10^{\circ} \mathrm{C}$ above ambient (centre) to $9^{\circ} \mathrm{C}$ above ambient (edge).

$P_{i}$, the analytical solution Eq. (14) is obtained with coefficients

$$
\begin{gathered}
A=\theta_{0}-\left(\frac{H D}{\kappa_{0}}+1\right) \frac{\kappa_{0}}{H} B, \\
B=-\frac{1}{\left(\left(H D / \kappa_{0}\right)+2\right)} \frac{1}{\kappa_{0} L W} \sum_{i} I_{00}^{i} P_{i} \\
C_{m n}=-S_{m n} \frac{H \tanh \gamma_{m n} D+\kappa_{0} \gamma_{m n}}{H+\kappa_{0} \gamma_{m n} \tanh \gamma_{m n} D}
\end{gathered}
$$

$$
\begin{aligned}
S_{m n}= & \frac{H+\kappa_{0} \gamma_{m n} \tanh \gamma_{m n} D}{2 H \kappa_{0} \gamma_{m n}+\left(\kappa_{0}^{2} \gamma_{m n}^{2}+H^{2}\right) \tanh \gamma_{m n} D} \\
& \times \frac{-4}{L W\left(1+\delta_{m 0}\right)\left(1+\delta_{n 0}\right)} \sum_{i} I_{m n}^{i} P_{i},
\end{aligned}
$$

where $\kappa_{0}$ is the thermal conductivity of the substrate. 
The corresponding expression for the thermal resistance matrix, describing the temperature rise of the surface heating elements above $\theta_{0}$, is

$$
\begin{aligned}
R_{T H_{i j}}= & \frac{\left(\left(H D / \kappa_{0}\right)+1\right)}{\left(\left(H D / \kappa_{0}\right)+2\right)} \frac{1}{H L W} I_{00}^{j} \\
& +\sum_{m n}^{\prime} \frac{H \tanh \gamma_{m n} D+\kappa_{0} \gamma_{m n}}{2 H \kappa_{0} \gamma_{m n}+\left(\kappa_{0}^{2} \gamma_{m n}^{2}+H^{2}\right) \tanh \gamma_{m n} D} \\
& \times \frac{4}{L W\left(1+\delta_{m 0}\right)\left(1+\delta_{n 0}\right)} \frac{I_{m n}^{i} I_{m n}^{j}}{I_{00}^{i}}
\end{aligned}
$$

Based on these expressions, the simulation of Figure 11 shows the simulated reverse side of a $\mathrm{Cu}$ / FR-4 mount bearing a 3-stage balanced amplifier MMIC. The simulation used a two-level structure with thermal resistance matrices constructed for the partial $\mathrm{Cu}$ layer and the FR-4 mount and matched at the interface. (This construction was for illustrative purposes only. An analytical solution can be constructed directly for this configuration, consisting of a double Fourier series in each layer, with Fourier components fully matched by solution of a corresponding linear system.) Two-layer solutions of this type require imposition of the radiation boundary condition on the surfaces of both layers, so as to produce exact cancellation of 'fictitious' radiative and convective fluxes at the matched interfaces.

As the electro-thermal model currently contains no explicit description of external fluid flow, the surface flux parameters $H_{0, D}$ in the radiation boundary condition, Eq. (13), were used as a fitting parameter to experimental data (setting $-H_{0}=H_{D}=H$ ) [19]. Only the average temperature on the back of the $\mathrm{Cu}$ layer was matched to the substrate.

Figures 12 and 13 show the impact of parameter variation on the calculated solution. Removing the partial $\mathrm{Cu}$ layer, Figure 12, leads to pronounced localisation of the temperature profile. The $2 \mu \mathrm{m}$ thick $\mathrm{Cu}$ layer therefore acts as an efficient heat spreader on FR-4. Figure 13 shows the effect of altering the value of the FR-4 thermal conductivity to that of AlN (three orders of magnitude higher).
The high AlN conductivity produces an almost uniform temperature across the surface of the mount.

\subsubsection{External Fluid Flow}

The need to use the heat transfer coefficient $H_{0, D}$ as a free parameter, limits the physicality of the description provided by the coupled electrothermal model in the case of large area surface losses. For larger area substrates $H_{0, D}$ can be obtained using standard convection correlations from the literature [11]. However, for the description to be fully physical $H_{0, D}$ must be obtained from a physical model. The full 3-dimensional coupled conduction-convection problem is complex, e.g. [60], however for simple cases of interest, such as laminar flow over a vertical plate, analytical solutions can be obtained for temperature distributions along the plate [61]. This offers the possibility of fully physical thermal resistance matrix calculations to make preliminary studies of external convection on device and circuit performance.

\subsection{Time Dependent Temperature}

The thermal resistance matrix approach described so far applies only to the temperature steady-state. However, the method generalises readily to treat the thermal time-dependent case in Laplace transform $s$-space $[19,24]$. Analytically generated thermal impedance matrices, describing thermal transients and capacitances, can be used directly in $s$-space, for instance in harmonic balance simulation, or in the time-domain where they correspond to the impulse response of the thermal system.

In coupled electro-thermal simulations thermal impedance matrices have to be calculated at a small number of points in s-space. A modest amount of precomputation then determines all thermal time evolution information. The coupled electro-thermal solution requires generalisation of 
the double Fourier series approach to treat arbitrary initial conditions, allowing repeated resetting of initial conditions in a time-stepping solution. (This generalisation also provides a double Fourier series treatment of volume heat sources and sinks, without the use of Green's function techniques.) The time-dependent coupled electro-thermal solution then reduces to a series of small non linear problems with order given by a small multiple of the number of active device elements.

The resulting formulation provides a fully coupled, physical electro-thermal solution in which the main approximations are flux and temperature discretisation at interfaces, interpolation of electrical power dissipation between discrete time-points, and numerical inversion of Laplace transforms. All surface and material non linearities, including temperature dependent diffusivity, are fully treated. This solution scheme compares favourably with time-stepping finite difference or finite element solutions, capable of comparable levels of detail and accuracy, which must iterate electrical and thermal physical solutions at each time step.

\section{SUMMARY AND CONCLUSIONS}

The problem of physical, coupled electro-thermal modelling for electro-thermal design of high power devices and circuits has been introduced. The combination of the quasi-2D Leeds Physical Model with the analytical thermal resistance matrix approach has been proposed as a viable solution. The Leeds Physical Model has been described and the details of the coupled electrothermal problem outlined. The motivation for the thermal resistance matrix approach to the thermal steady-state case has been presented.

The most general formulation of the resistance matrix approach offers a potentially competitive solution scheme for any physical system, described by an equation in some complex volume, which can be resolved into subsystems allowing linear analytical solution with specified boundary conditions. The resistance matrix approach then amounts to matching of field, $\psi$, and its derivative, $\psi^{\prime}$, at subsystem interfaces, with the resistance matrix relating linearly $\psi$ and $\psi^{\prime}$ at distinct interfaces of any given subvolume. This approach produces a minimal system to be solved for (discretised) interface unknowns, thus providing full 3-dimensional solutions for the whole complex volume. It is of particular value for describing distinct physical systems coupled in a relatively small number of isolated regions.

Applying this approach to the coupled electrothermal system, the construction of thermal resistance matrices has been described for the case of the homogeneous MMIC, bearing an arbitrary distribution of power transistor fingers. It was shown that this construction leads to a small non linear problem for the coupled electrical description, readily soluble by Newton methods. Construction of resistance matrices was then outlined for multi-layer MMICs and MMICs with superstructure such as surface metallisation and air bridges. Constructions were presented for MMICs with vias and with partial substrate thinning, and the application of the radiation boundary condition for larger area substrates was described.

The steady-state thermal resistance matrix approach described here, only requires construction of the global thermal resistance matrix for a complex 3-dimensional system once, prior to the coupled electro-thermal simulation. Precomputation to construct the thermal resistance matrices is modest, as matrices for thermal subsystems are given by simple analytical expressions. The global thermal resistance matrix is obtained by simple matrix manipulations on the subsystem matrices, to impose temperature and flux matching. Construction time for the thermal resistance matrices has no impact on coupled electro-thermal run time. The magnitude of the non linear coupled electrical problems to be solved self-consistently is typically given by the number of active device elements, not determined by the internal complexity of the device structure. 
Full treatment of thermal non linearities has been described, including treatment of temperature dependent conductivity (perhaps requiring non linear interface matching) and non linear surface fluxes. Full treatment of interface matching and surface flux non linearities, based on the linear thermal resistance matrix approach, alters the size of the corresponding coupled problem, but the size of the non linear system to be solved can be minimised by careful choice of surface elementary areas, to take full account of expected temperature distributions and minimise the degree of surface discretisation.

The combination of the Leeds Physical Model and the analytical thermal resistance matrix approach offers order of magnitude speed up on coupled electro-thermal solutions based on numerically intensive electrical and thermal solutions, capable of comparable levels of detail and accuracy. The fully physical model will provide a predictive tool for electro-thermal design studies on CAD timescales.

\section{Acknowledgements}

This work was supported by the U.S. Army Research Office through Clemson University as a Multidisciplinary Research Initiative on QuasiOptics, agreement Number DAAG55-97-K-0132.

\section{References}

[1] Bonani, F., Ghione, G., Pirola, M. and Naldi, C. U. (1994). "A large-scale, self-consistent thermal simulator for the layout optimization of power III-V field-effect and bipolar transistors", IEEE GaAs '94, Proc. European Gallium Arsenide and Related III-V Compounds Applications Symp., pp. 411-414.

[2] Bonani, F., Ghione, G., Pirola, M. and Naldi, C. U. (1994). "Large-scale, computer-aided thermal design of power GaAs integrated devices and circuits", Proc. IEEE GaAs IC Symposium, pp. 141-144.

[3] Bonani, F., Ghione, G., Pirola, M. and Naldi, C. U. (1995). "Thermal CAD for power III-V devices and MMICs", Proc. SBMO/IEEE MTT-S Internat. Microwave and Optoelectronics Conf., 1, 352-357.

[4] Webb, P. W. and Russell, I. A. D. (1989). "Thermal resistance of gallium-arsenide field-effect transistors", $I E E$ Proc., 136, Pt. G, No. 5, 229-234.
[5] Webb, P. W. and Russell, I. A. D. (1991). "Thermal simulation of transients in microwave devices", IEE Proc., 138, Pt. G, No. 3, 329-334.

[6] Gui, X., Webb, P. W. and Gao, G.-B. (1992). "Use of the three-dimensional TLM method in the thermal simulation and design of semiconductor devices", Trans. Electron Dev., 39(6), 1295-1302.

[7] Webb, P. W. (1993). "Thermal modeling of power gallium arsenide microwave integrated circuits", IEEE Trans. Electron Dev., 40(5), 867-877.

[8] Webb, P. W. (1997). "Thermal design of gallium arsenide MESFETs for microwave power amplifiers", IEE Proc. Circuits Devices Syst., 144(1), 45-50.

[9] Dorkel, J.-M., Tounsi, P. and Leturcq, P. (1996). "Threedimensional thermal modeling based on the two-port network theory for hybrid or monolithic integrated power circuits", IEEE Trans. Components, Packaging and Manufacturing Technology-Part A, 19(4), 501-507.

[10] Ellison, G. N. (1996). "Thermal analysis of circuit boards and microelectronic components using an analytical solution to the heat conduction equation", Proc. 12th Annual IEEE Semiconductor Thermal Measurement and Management Symp., pp. 144-150.

[11] Ellison, G. N. (1995). "Thermal analysis of microelectric packages and printed circuit boards using an analytic solution to the heat conduction equation", Advances in Engineering Software, 22(2), 99-111.

[12] Anholt, R., Electrical and thermal characterization of MESFETs, HEMTs, and HBTs, Boston: Artech House, 1994.

[13] Anholt, R. (1998). "HBT thermal element design using an electro/thermal simulator", Solid-State Electronics, 42(5), $857-864$

[14] Anholt, R. (1998). "Thermal impedances of multi-finger heterojunction bipolar transistors", Solid-State Electronics, 42(5), 865-869.

[15] Anholt, R. (1998). "FET and HEMT thermal impedances", Solid-State Electronics, 42(5), 849-855.

[16] Ghione, G. and Naldi, C. U. (1989). "A self-consistent thermal simulator of MMIC multi-gate GaAs active devices", ESPRIT'89, Proc. 6th Annual ESPRIT Conf. (EUR 12512), Brussels, Belgium, pp. 73-88.

[17] Snowden, C. M. (1997). "Large-signal microwave characterization of AlGaAs/GaAs HBT's based on a physicsbased electrothermal model", IEEE Trans. Micro. Theory and Tech., 45(1), 58-71.

[18] Batty, W., Panks, A. J. and Snowden, C. M. (1999). "Fully coupled electro-thermal simulation of MMICs and MMIC arrays based on a physical model", IEEE MTT-S Internat. Microwave Symp. Dig., 2, Paper TU4C-5, 693-696.

[19] Batty, W., Panks, A. J., Johnson, R. G. and Snowden, C. M. (1999). "Electro-thermal modelling and measurement for spatial power combining at millimeter wavelengths", IEEE Trans. Microwave Theory Techniques, 47(12), $2574-2585$.

[20] Cheng, Y.-K., Teng, C.-C., Dharchoudhury, A., Rosenbaum, E. and Kang, S.-M., "A chip-level electrothermal simulator for temperature profile estimation of CMOS VLSI chips", Proc. IEEE Int. Symp. Circuits and Systems, 4, 580-583, Atlanta, 1996.

[21] Curtice, W. R. and Yun, Y.-H. (1981). "A temperature model for the GaAs MESFET", IEEE Trans. Electron Dev., ED-28(8), 954-962.

[22] Johnson, R. G., Snowden, C. M. and Pollard, R. D. (1997). "A physics-based electro-thermal model for 
microwave and millimetre wave HEMTs", IEEE MTT-S Internat. Microwave Symp. Dig., 3, Paper TH3E-6, $1485-1488$.

[23] Albasha, L., Johnson, R. G., Snowden, C. M. and Pollard, R. D., "An investigation of breakdown in power HEMTs and MESFETs utilising an advanced temperature-dependent physical model", Proc. IEEE 24th Internat. Symp. Compound Semiconductors, pp. 471-474, San Diego, 1997.

[24] Batty, W., Panks, A. J., Johnson, R. G., and Snowden, C. M. "Electro-thermal physical modelling and measurement of MMICs and MMIC grid arrays with thermal transients due to turn-on and pulsed operation", IEEE Trans. Microwave Theory Techniques, to be submitted.

[25] Baureis, P., "Electrothermal modeling of multi-emitterheterojunction bipolar transistors (HBT's)", Proc. INMMC-94, pp. 145-148, Duisburg, Germany, 1994.

[26] Liou, L. L., Ebel, J. L. and Huang, C. I. (1993). "Thermal effects on the characteristics of $\mathrm{AlGaAs} / \mathrm{GaAs}$ heterojunction bipolar transistors using two-dimensional numerical simulation", Trans. Electron Dev., 40(1), 35-43.

[27] Joy, R. C. and Schlig, E. S. (1970). "Thermal properties of very fast transistors", IEEE Trans. Electron Dev., ED17(8), 586- 594.

[28] Kokkas, A. G. (1974). "Thermal analysis of multiple-layer structures", IEEE Trans. Electron Dev., ED-21(11), 674-681.

[29] Leturcq, P., Dorkel, J.-M., Napieralski, A. and Lachiver, E. (1987). "A new approach to thermal analysis of power devices", IEEE Trans. Electron Dev., ED-34(5), $1147-1156$.

[30] Wachutka, G. (1989). "An extended thermodynamic model for the simultaneous simulation of the thermal and electrical behaviour of semiconductor devices", Proc. 6th Internat. NASECODE Conf., pp. 409-414.

[31] Gao, G.-B., Wang, M.-Z., Gui, X. and Morkoc, H. (1989). "Thermal design studies of high-power heterojunction bipolar transistors", IEEE Trans. Electron Dev., 36(5), 854-863.

[32] Fan, M. S., Christou, A. and Pecht, M. G. (1992). "Twodimensional thermal modeling of power monolithic microwave integrated circuits (MMIC's)", IEEE Trans. Electron Dev., 39(5), 1075-1079.

[33] Higgins, J. A. (1993). "Thermal properties of power HBT's", IEEE Trans. Electron Dev., 40(12), 2171-2177.

[34] Liou, L. L. and Bayraktaroglu, B. (1994). "Thermal stability analysis of $\mathrm{AlGaAs} / \mathrm{GaAs}$ heterojunction bipolar transistors with multiple emitter fingers", IEEE Trans. Electron Dev., 41(5), 629-636.

[35] Ishizuka, M., Hisano, K., Sasaki, T. and Fukuoka, Y., "Application of the thermal network method to the transient thermal analysis of multichip modules", Proc. Japan Int. Symp. Electronic Manufacturing Technology, Paper WC1-4, pp. 464-467, Omiya, 1995.

[36] Lai, J. and Majumdar, A. (1996). "Concurrent thermal and electrical modeling of sub-micrometer silicon devices", J. Appl. Phys., 79(9), 7357-7361.

[37] Lu, K. and Snowden, C. M. (1996). "Analysis of thermal instability in multi-finger power AlGaAs/GaAs HBT"s", IEEE Trans. Electron Dev., ED-43(11), 1799-1805.

[38] Digele, G., Lindenkreuz, S. and Kasper, E. (1997). "Fully coupled dynamic electro-thermal simulation", IEEE Trans. Very Large Scale Integration (VLSI) Systems, 5(3), 250-257.

[39] Digele, G., Lindenkreuz, S. and Kasper, E., "Electrothermal interaction on circuit level under the influence of packaging", Proc. 27th European Conf. Solid-State Device Research, pp. 460-463, Stuttgart, 1997.

[40] Snowden, C. M., "Characterization and modelling of microwave and millimeter-wave large-signal device-circuit interaction based on electro-thermal physical models", IEEE MTT-S Internat. Microwave Symp. Dig., 1, Paper TU3B-2, 155-158, Colorado, 1997.

[41] Breglio, G., Pica, S. and Spirito, P., "Thermal effects and electro-thermal modeling in power bipolar transistors", Proc. 21st Internat. Conf. Microelectronics (MIEL'97), 1, 383-390, Yugoslavia, 1997.

[42] Batty, W., Johnson, R. G. and Snowden, C. M., "Electrothermal HEMT modelling for microwave and millimeter wave Circuit Design", Proc. IOP Condensed Matter and Materials Physics Conference, Paper SSb.P2.15, p. 133, Exeter, 1997.

[43] Morton, C. G. and Snowden, C. M., "MESFET and HEMT design using fast physical device simulation", Microwave Journal, pp. 94-113, September, 1998.

[44] Souverain, P., Camps, T., Faleh, M. S., Cazarre, A., Tasselli, J., Marty, A. and Bailbe, J. P. (1998). "Threedimensional modelling of the heat flow into a GaAs substrate. Influence of thermal phenomena on the RF behaviour of power HBTs and technological optimization", Microelectron. Reliab., 38(4), 553-557.

[45] Szekely, V., Rencz, M. and Courtois, B., "Tracing the thermal behaviour of ICs", IEEE Design Test Computers, pp. 14-21, April-June, 1998.

[46] Snowden, C. M., "Electrothermal transistor models for large-signal design", IEEE MTT-S Workshop Digest on "New developments in time domain methods for non-linear design', pp. 1-16, June, 1998.

[47] Snowden, C. M., "Electro-thermal microwave transistor models for large-signal CAD", Proc. Integrated Nonlinear Microwave and Millimeterwave Circuits Conf., pp. 56-68, Duisburg, October, 1998.

[48] Snowden, C. M., "Non-linear microwave CAD using physics-based active device models", Proc. Asia Pacific Microwave Conf., pp. 1417-1424, Yokahama, Japan, 1998.

[49] Snowden, C. M. and Pantoja, R. R. (1989). "Quasi-twodimensional MESFET simulations for CAD", IEEE Trans. Electron Dev., 36(9), Pt. 1, 1564-1574.

[50] Morton, C. G., Atherton, J. S., Snowden, C. M., Pollard, R. D. and Howes, M. J. (1996). "A large-signal physical HEMT model", IEEE MTT-S Internat. Microwave Symp. Dig., pp. 1759-1762.

[51] Morton, C. G. and Snowden, C. M. (1998). "Comparison of quasi-2D and ensemble Monte Carlo simulations for deep submicron HEMTs", IEEE MTT-S Internat. Microwave Symp. Dig., 1, 153-156.

[52] Blakemore, J. S. (1982). "Semiconducting and other major properties of gallium arsenide", J. Appl. Phys., 53, R123-186.

[53] Panks, A. J. (1997). "The synthesis of two-terminal negative conductances based on physical modelling", Ph.D. Thesis, University of Leeds.

[54] York, R. A. and Popović, Z. B. Eds., Active and quasioptical arrays for solid-state power combining, Wiley (1997).

[55] Joyce, W. B. (1975). "Thermal resistance of heat sinks with temperature-dependent conductivity", Solid-State Electronics, 18, 321-322.

[56] Maycock, P. D. (1967). "Thermal conductivity of silicon, germanium, III-V compounds and III-V alloys", SolidState Electronics, 10, 161-168. 
[57] Bonani, F. and Ghione, G. (1995). "On the application of the Kirchhoff transformation to the steady-state thermal analysis of semiconductor devices with temperaturedependent and piecewise inhomogeneous thermal conductivity", Solid-State Electronics, 38(7), 1409-1412.

[58] Brewster, R. A. and Sherif, R. A. (1992). "Thermal analysis of a substrate with power dissipation in the vias", IEEE Trans. Components, Hybrids, Manufacturing Technology, 15(5), 667-674.

[59] Goldberg, J. L. (1972). Systems of Ordinary Differential Equations.

[60] Liu, Y., Phan-Thien, N., Kemp, R. and Luo, X.-L. (1997). "Three-dimensional coupled conduction-convection problem for three chips mounted on a substrate in an enclosure", Numerical Heat Transfer, Part A, 32, 149-167.

[61] Lee, S. and Yovanovich, M. M. (1992). "Linearization of natural convection from a vertical plate with arbitrary heat-flux distributions", Trans. ASME Journal of Heat Transfer, 114, 909-916.

\section{Authors' Biographies}

William Batty was born in England in 1963. He received the B.A. degree in physics from Oxford University, Oxford, U.K., in 1985 and the Ph.D. degree from Surrey University, Guildford, U.K., in 1990. He has worked as a Research Fellow at the University of York (1991 - 1996), University of Wales, Bangor (1996-1997), and the University of Leeds (1997-present). His research has spanned the whole frequency spectrum from optoelectronic quantum well device modelling, particularly bipolar diode lasers and quantum-confined Stark effect optical modulators, through $\mathrm{THz}$ unipolar intersubband lasers, to his current work on electrothermal effects in MMICs and MMIC grid arrays for spatial power combining at millimeter wavelengths.

Andrew J. Panks received the B.Eng. and Ph.D. degrees in Electronic and Electrical Engineering from the University of Leeds, UK, in 1993 and 1997 respectively. Since 1997 he has worked as a Research Fellow, initially with the Microwave and Terahertz Technology Group and more recently with the Institute of Microwaves and Photonics at Leeds University. His research interests include large signal modelling of nonlinear circuits, physical device modelling, thermal modelling and measurements of passive and active microwave circuits.

Robert G. Johnson was born in Leeds, England in 1969. He obtained a B.A. in Mathematics from Cambridge University, UK, in 1991, an M.Sc. in Nonlinear Maths from the University of Bath in 1992 and a Ph.D. from the University of Leeds in 1995. His current research interests are in electro-thermal modelling of HEMTs using quasitwo dimensional physical methods for CAD applications.

Christopher M. Snowden received the B.Sc. (Hons.), M.Sc. and Ph.D. degrees from the University of Leeds, England. He is currently Executive Director of Technology of Filtronic plc and Professor of Microwave Engineering at the University of Leeds. He is a Fellow of the IEEE, Fellow of the IEE and a Chartered Engineer. He was awarded the 1999 Microwave Prize of the IEEE Microwave Theory and Techniques Society. His main research interests include semiconductor device and circuit modelling (CAD), microwave, millimetre-wave and optoelectronic circuit technology, and microwave, terahertz and optical nonlinear subsystem design. $\mathrm{He}$ has written 8 books, over 200 refereed journal and conference papers, and many other articles. 

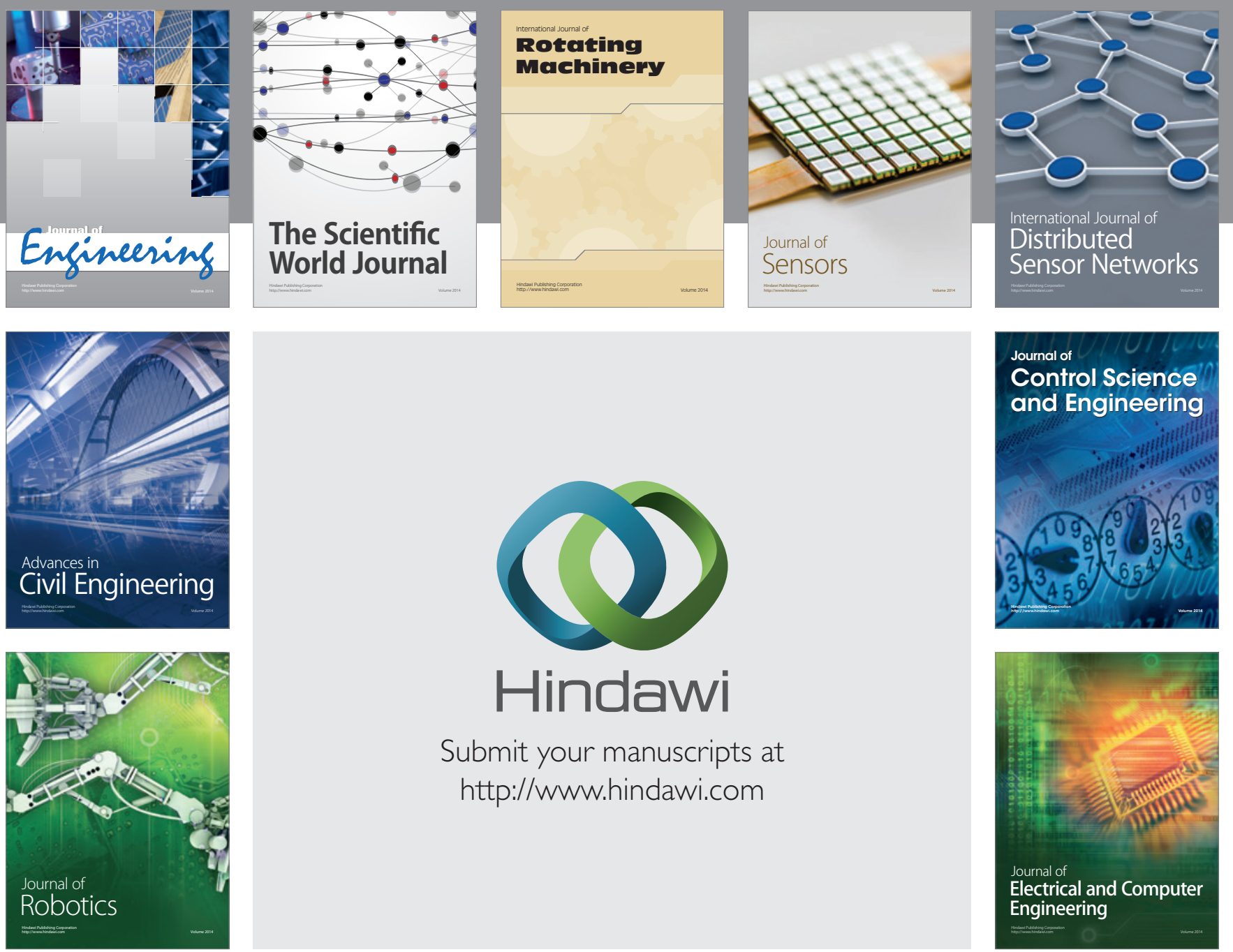

Submit your manuscripts at

http://www.hindawi.com
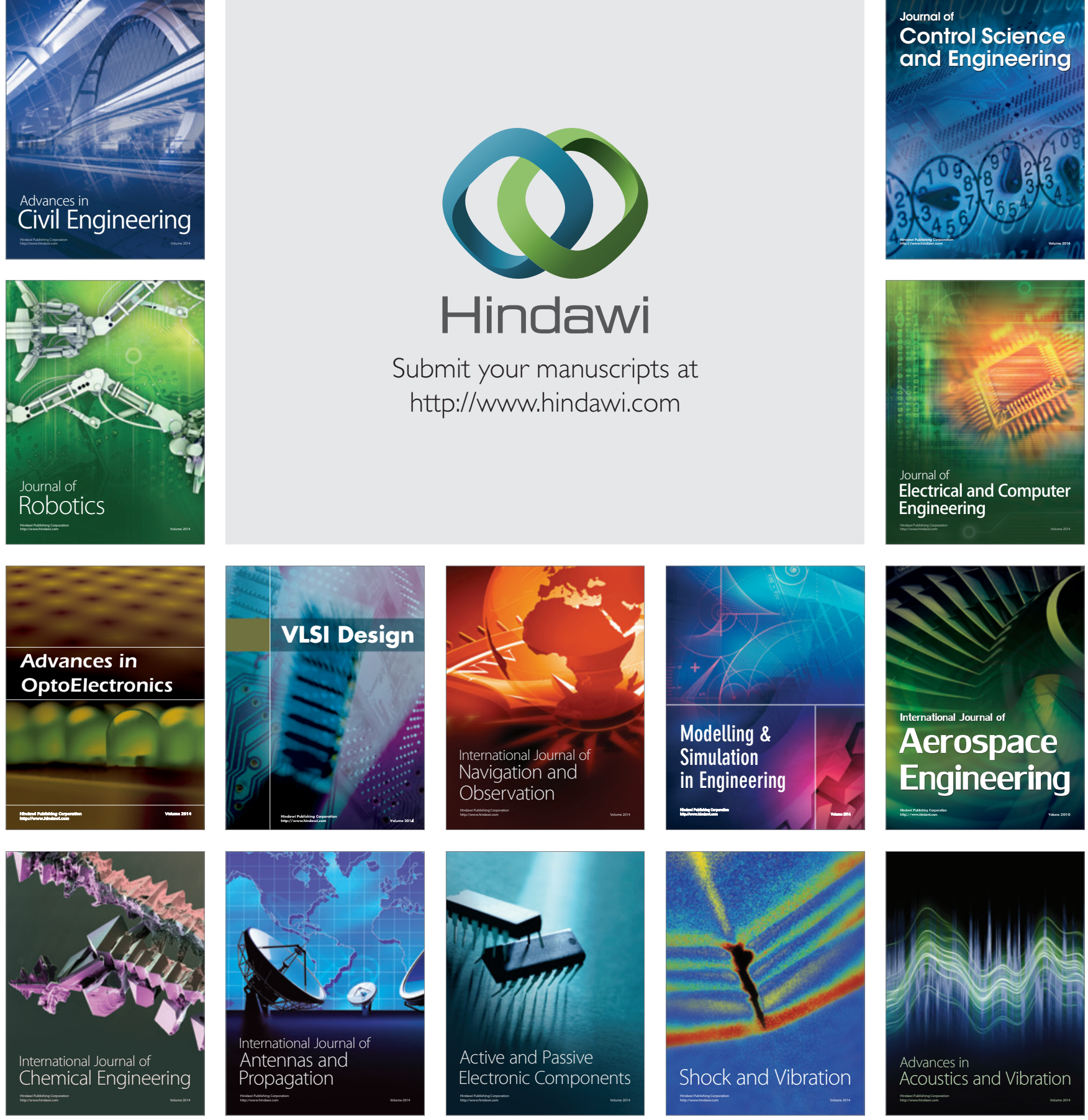